\title{
Exkurs
}

\author{
Hans-Peter Blossfeld \& Hans-Günther Roßbach
}

\section{Neue Herausforderungen für die Kindertagesstätten: Professionalisierung des Personals in der Frühpädagogik}

\author{
New challenges for pre-school day-care institutions: Professionalization of \\ personnel in early childhood education
}

\section{Zusammenfassung:}

Die meisten Experten sind sich heute weitgehend einig, dass das entscheidende Fundament für spätere erfolgreiche Bildungs- und Berufskarrieren im Lebenslauf bereits in der frühkindlichen Entwicklung gelegt wird. Qualitativ hochwertige Bildungsangebote in den Kindertagesstätten sind deswegen auch ein wichtiges Ziel. Allen Kindern muss dort ein Zugang zu vielfältigen Bildungsangeboten und Lerninhalten eröffnet werden. Eine Schlüsselrolle kommt in diesem Zusammenhang der Qualität des frühpädagogischen Personals zu. Der vorliegende Beitrag fasst die Ergebnisse des Gutachtens des Aktionsrats Bildung aus dem Frühjahr 2012 zur Professionalisierung des pädagogischen Personals in der frühkindlichen Bildung zusammen. Er skizziert zunächst die gestiegenen gesellschaftlichen Erwartungen und Anforderungen an die frühpädagogischen Einrichtungen als Bildungseinrichtungen. Dann diskutiert er die Rolle der Qualität frühpädagogischer Einrichtungen. Dabei zeigt sich, dass dort die entscheidenden Qualitätsmerkmale das Ausbildungsniveau und die Kompetenzen des frühpädagogischen Personals sind. Der Beitrag fragt auch, wer auf welcher Ebene ausgebildet wird und welche Ausbildungskapazitäten dem Personalbedarf gegenüberstehen. Dabei stellt sich heraus, dass das frühpädagogische Personal noch auf längere Zeit sowohl auf Fachschul- als auch auf Hochschulebene ausgebildet werden muss. Auf beiden Ebenen werden im vorliegenden Beitrag Handlungsbedarfe für die Politik identifiziert, wobei auch die

\section{Summary:}

Most experts would agree that early education is an important foundation for later school achievements and employment prospects over the life course. One way of achieving this goal is to provide high quality education in the early years which gives young children access to diverse learning opportunities. The practitioners in early childhood institutions play a key role in this process. This paper summarizes the most important recommendations of a 2012 report published by Aktionsrat Bildung (Action Counsel for Education) on the professionalization of educators in early childhood institutions. Following a brief outline of the increased societal expectations in terms of the educational role of early childhood provision, a research review focuses on issues of quality. The formal level of staff qualification and staff competences are shown to be central dimensions of overall quality. Relating these research findings to the situation in Germany, the paper asks what kind of professional workers are needed in early childhood institutions, and at what level and with what particular focus these practitioners should be educated and trained, bearing in mind the current capacities. It seems that in the intermediate future, the majority of personnel will continue to be trained at vocational school level, with a (growing) minority following a higher education course of studies. The paper identifies priorities for policy makers relating to these different levels of initial training and to the continuing professional development of the early years 
Fort- und Weiterbildung mit einbezogen wird.

Schlagwörter: Professionalisierung, Qualität des pädagogischen Personals, frühkindliche Erziehung und Bildung workforce.

Key words: professionalization, educational staff qualification, early childhood education

\section{Einleitung}

Erziehung, Bildung und Betreuung sind schon seit Langem die Funktionen von Kindertageseinrichtungen in Deutschland. Allerdings haben sich die Gewichte innerhalb dieser Trias in der Geschichte von Kindertagesrichtungen immer wieder mal verschoben (vgl. Roßbach 2008). In den vergangenen Jahren hat sich - nicht zuletzt angestoßen durch die Diskussion um das relativ schlechte Abschneiden deutscher Schülerinnen und Schüler bei internationalen Schulleistungsvergleichsstudien wie IGLU, TIMMS oder PISA - die öffentliche Erwartung an frühpädagogische Einrichtungen wieder stärker der Bildungsfunktion zugewandt. Kindertageseinrichtungen - speziell für Kinder ab dem dritten Lebensjahr - werden nicht mehr vorrangig als Betreuungseinrichtungen betrachtet, sondern als zentrale Bildungseinrichtungen wahrgenommen, die ihre Aufgaben nur erfüllen können, wenn ihre Arbeit von hoher Qualität ist. Besondere Aufmerksamkeit erfährt dabei die Förderung von Kindern aus bildungsfernen Schichten und aus Familien mit Migrationshintergrund.

Je stärker sich die Kindertagesstätten von reinen Betreuungseinrichtungen in vorschulische Systeme verändern, desto bedeutsamer ist die Qualifizierung des frühpädagogischen Personals. In der Vergangenheit wurde die Ausbildung des frühpädagogischen Personals vor allem am Typus der Betreuungsinstitution orientiert. Diese Akzentuierung lässt sich heute nicht mehr halten. Die Qualifikationen des frühpädagogischen Personals, seine Differenzierung in den Einrichtungen und die Personalentwicklung der vorhandenen Mitarbeiter sind Gegenstand des vorliegenden Aufsatzes. Er fasst die Grundideen und Vorschläge des neuen Gutachtens des Aktionsrats Bildung (2012) zur Professionalisierung in der Frühpädagogik kurz zusammen.

Dabei werden in einem ersten Schritt die gesellschaftlichen Erwartungen an die frühpädagogischen Einrichtungen als Bildungseinrichtungen skizziert, und es wird auf der Grundlage von neueren Forschungsbefunden diskutiert, welchen Beitrag der Besuch und vor allem die Qualität der frühpädagogischen Einrichtungen leisten können. Besonders wichtige Merkmale der Qualität sind dabei das Ausbildungsniveau und die Kompetenzen des frühpädagogischen Personals. In einem zweiten Schritt wird dann die gegenwärtige Ausbildungssituation beleuchtet. Es wird danach gefragt, wer auf welcher Ebene ausgebildet wird und welche Ausbildungskapazitäten dem bis zum Jahr 2020 entstehenden Personalbedarf gegenüberstehen.

Die Begriffe frühpädagogische Einrichtung und Kindertageseinrichtung werden in diesem Beitrag synonym verwendet und umfassen Krippen, Kindergärten, Horte für die außerschulische Betreuung von Schulkindern und altersgemischte Einrichtungen. Eine Differenzierung entsprechend der verschiedenen Altersgruppen im frühpädagogischen Bereich ist in vielerlei Hinsicht sinnvoll. Oftmals wird unterschieden zwischen der Arbeit 
mit Kindern unter drei Jahren und mit Kindern von drei Jahren bis zum Schulbeginn. Allerdings verschwimmt diese Grenze gegenwärtig, da „traditionelle“ Kindergärten zum einen Krippengruppen aufnehmen und zum anderen vermehrt unter dreijährige Kinder (z.B. ab dem vollendeten zweiten Lebensjahr) in die Gruppen integriert werden, die bis vor Kurzem noch für Kinder ab dem vollendeten dritten Lebensjahr vorgesehen waren. $\mathrm{Zu}$ dem gibt es in einigen Bundesländern ausgeprägte Tendenzen zu einer größeren Altersmischung in Kindertagesstätten, die dann z.B. neben getrennten Hortgruppen für die außerschulische Betreuung und Bildung von (Grund-)Schulkindern auch Gruppen für Kinder in der gesamten Altersspanne von null bis zu ca. zehn Jahren umfassen.

Viele Problemstellungen betreffen die verschiedenen Altersgruppen grundsätzlich gleichermaßen, sind aber im Hinblick auf die Bildungsförderung und den Ausgleich von sozial bedingten Disparitäten für unter dreijährige Kinder von besonderer Brisanz: Eine frühe Sprachförderung von unter dreijährigen Kindern, vor allem von Kindern, deren Herkunftssprache nicht Deutsch ist, stellt beispielsweise besondere Anforderungen an wissenschaftlich abgesicherte Kompetenzen in den Bereichen Sprachdiagnostik und Früherkennung. Dies betrifft ebenso die Förderung in den Kindertageseinrichtungen sowie den möglichen Verweis an Einrichtungen, die therapeutische Maßnahmen anbieten. Ähnliches gilt auch für andere Förderbereiche, sodass Empfehlungen für die Professionalisierung des Personals hinsichtlich der Erziehung, Bildung und Betreuung von unter dreijährigen und älteren Kindern nicht zu sehr auseinanderdriften. Folglich wird hier allgemein von frühpädagogischen Einrichtungen und Kindertagesstätten gesprochen, ohne auf weitere Altersdifferenzierungen einzugehen.

\section{Veränderte gesellschaftliche Erwartungen an frühpädagogische Einrichtungen}

Die öffentliche Diskussion in der „Nach-PISA-Zeit“ hat zu vielfältigen und oftmals unkoordinierten Reformbestrebungen im deutschen Bildungswesen geführt. In diese Bemühungen wurde zunehmend auch der vorschulische Bereich einbezogen (vgl. Roßbach 2004). Unter dem Motto „Auf den Anfang kommt es an!“ gewann dieser in den letzten Jahren erneut die öffentliche Aufmerksamkeit, die ihm schon einmal während der Bildungsreform der 1960er und 1970er Jahre zukam. Um die Bildungsfunktion in der Trias von Erziehung, Bildung und Betreuung zu betonen und um $\mathrm{zu}$ mehr Verbindlichkeit in der pädagogischen Arbeit zu kommen, haben alle Bundesländer in den letzten Jahren Bildungspläne für den frühpädagogischen Bereich entwickelt (vgl. Diskowski 2008). Viel stärker als noch vor einem Jahrzehnt wird neben einer breiten Förderung der Persönlichkeit der Kinder die Förderung in spezifischen Inhaltsbereichen wie Mathematik, Sprache, Vorläuferfähigkeiten für Lese- und Rechtschreibkompetenzen oder Naturwissenschaften betont. Die Bildungspläne heben hervor, dass dies nicht (nur) in spezifischen, d.h. in vorgeplanten und vorstrukturierten, Angeboten geschehen soll, sondern alltagsintegriert und somit eingebettet in die üblichen Alltagstätigkeiten in einer frühpädagogischen Einrichtung. Neue Anforderungen werden zudem an eine (sehr) frühe Sprachdiagnostik und Sprachförderung gestellt, um Kindern mit Defiziten in der altersgemäßen Beherrschung der deutschen Sprache einen guten Start ihrer Schullaufbahn zu ermöglichen. Vor allem 
Kinder mit einer anderen Herkunftssprache als Deutsch sollen von dieser Art der Förderung profitieren. Eine weitere Herausforderung stellt die Bewältigung der heterogener gewordenen Zusammensetzung der Kindergruppen in den frühpädagogischen Einrichtungen dar. Dies gilt vor allem für die zunehmend flexibleren Altersgrenzen der Kinder bei Eintritt in frühpädagogische Einrichtungen und im Besonderen für Kinder mit Migrationshintergrund. Die Heterogenität wird noch verstärkt durch die Forderung nach Inklusion von Kindern mit besonderem Förderbedarf. Zudem werden von den frühpädagogischen Einrichtungen eine Erweiterung der Elternarbeit und eine Weiterentwicklung der Kindertageseinrichtungen zu Familienzentren erwartet. Diese sollen die Erziehung, Bildung und Betreuung der Kinder mit Familienbildung sowie mit weiteren Hilfen für die Familien und Kinder verbinden. Schließlich soll der Besuch der frühpädagogischen Einrichtungen einen spezifischen Beitrag zur Schulvorbereitung leisten. Hier muss allerdings vor einem Fehlschluss gewarnt werden (vgl. Kluczniok/Roßbach/Große 2010; Roßbach/Sechtig/ Freund 2010): Eine stärker ,schulvorbereitend“ orientierte Förderung ist nicht gleichbedeutend mit einer Vorwegnahme von Schule und einer Anleitung im Sinne einer schulischen Unterweisung. Es stellt sich sogar die Frage, ob „schulisch“ orientierte Vermittlungsformen - im Sinne von lehrgangsförmigem Unterricht - für Kinder im vorschulischen Alter überhaupt Erfolg versprechend sind, da spezifische entwicklungspsychologische Voraussetzungen in diesem Alter noch nicht vollständig vorliegen. Besonders bedeutsam sind hier Entwicklungsprozesse im Arbeitsgedächtnis, d.h. die Entwicklung der automatisierten Aktivierung des inneren Nachsprechens zwischen dem fünften und dem sechsten Geburtstag, die als eine Voraussetzung für den systematischen Erwerb von Schriftsprache und arithmetischen Fertigkeiten - wie er in einer Vielzahl von didaktischen Konzepten für die Grundschule vorgesehen ist - zu betrachten ist (vgl. Hasselhorn 2005; Hasselhorn/Grube 2008). Außerdem zeigen nationale und internationale Beispiele, dass eine frühe Förderung von Vorläuferfähigkeiten der später in der Schule erforderlichen Kompetenzen erfolgreich sein kann, wenn Fördermaßnahmen in die üblichen spielerischen Aktivitäten im Kindergarten eingebettet sind.

Neben verstärkten Bildungserwartungen an die frühpädagogischen Einrichtungen bestimmt auch der deutliche quantitative Ausbau des frühpädagogischen Bereichs die gegenwärtige Diskussion. Ab dem 1. August 2013 hat jede Familie mit einem Kind ab dem vollendeten ersten Lebensjahr das Recht auf einen Betreuungsplatz. Das angestrebte Ziel ist ein Betreuungsanteil von 35 Prozent der ein- bis dreijährigen Kinder. Je nach Schätzung müssen im Zeitraum von heute bis August 2013 dafür ca. 300.000 Plätze in Kindertageseinrichtungen und Kindertagespflege geschaffen werden (vgl. Schilling 2011a). Ebenso sollen das Angebot an Ganztagesbetreuungen im vorschulischen Bereich sowie die außerschulische Betreuung und Bildung von (Grund-)Schulkindern im Hort ausgeweitet werden. Während in den letzten Jahrzehnten in der Diskussion die Tendenz zu beobachten war, Betreuungsfunktionen gegen Erziehungs- und Bildungsfunktionen auszuspielen, werden die beiden Leistungsbereiche frühpädagogischer Einrichtungen heute als gleichberechtigt betrachtet.

Zusammenfassend lassen sich folgende drei Erwartungen festhalten: (1) Die frühpädagogischen Einrichtungen sollen alle Kinder optimal fördern und auf ihre zukünftige Schulkarriere vorbereiten. Der Bildungsauftrag beschränkt sich dabei nicht auf eng umrissene kognitive Kompetenzen, sondern ist eingebettet in eine breitere Persönlichkeits- 
bildung der Kinder. (2) Die frühpädagogischen Einrichtungen sollen insbesondere Defizite bei Kindern aus benachteiligten Familien reduzieren und so zu mehr sozialer Gerechtigkeit und einer Reduzierung von sozial bedingten Ungleichheiten noch vor Schulbeginn beitragen. (3) Sie sollen einen zentralen Beitrag zur Vereinbarkeit von Familien- und Berufstätigkeit insbesondere von Frauen bzw. Müttern leisten.

Damit die verschiedenen Erwartungen erfüllt werden können, stellen sich heute veränderte Anforderungen an die Quantität und Qualität des pädagogischen Personals. Zum einen wird für den Ausbau an (ganztägigen) Betreuungsplätzen zusätzliches Personal benötigt; zum anderen reicht es aber nicht aus, den Personalbedarf primär durch Zusatzoder Hilfskräfte zu decken, die auf niedrigem Niveau ausgebildet sind. Vielmehr verlangen die skizzierten gestiegenen öffentlichen Erwartungen an die frühpädagogischen Einrichtungen hinreichend pädagogisch ausgebildetes Fachpersonal, da hier Aufgaben angesprochen werden, die in der Vergangenheit nicht bzw. nur teilweise in der fachschulischen Ausbildung berücksichtigt wurden (z.B. im Hinblick auf den produktiven Umgang mit Bildungsplänen, die gezielte Förderung in spezifischen Bildungsbereichen, die frühe Sprachdiagnostik und Sprachförderung, vor allem für unter dreijährige Kinder, den Umgang mit Heterogenität und inklusiver Erziehung, Bildung und Betreuung, die Weiterentwicklung der Einrichtungen zu Familienzentren oder die Verbesserung des Übergangs in die Grundschule). Der quantitative Ausbau allein reicht also nicht aus, um die Bildungsziele zu erreichen; stattdessen bedarf es einer deutlichen Verbesserung der Qualität.

\section{Positive Auswirkungen frühpädagogischer Einrichtungen auf die Entwicklung der Kinder und auf ihre Familien}

Sicherlich ist die Erziehung, Bildung und Betreuung in frühpädagogischen Einrichtungen nur ein Element im Gesamt der familialen und institutionellen - speziell schulischen Einflüsse auf die Entwicklung und Bildung der Kinder. Daher sind realistische Erwartungen angeraten. Gleichwohl kann nach vorliegenden Studien von positiven Auswirkungen auf die Entwicklung der Kinder und auf ihre Familien ausgegangen werden. Dabei stellt sich der internationale Forschungsstand zusammengefasst wie folgt dar (vgl. ausführlicher die Überblicksartikel Roßbach 2005, 2011; Roßbach/Kluczniok/Kuger 2008).

Auswirkungen auf sozial-emotionale Kompetenzen von Kindern: Für Kinder unter drei Jahren finden sich verschiedene Hinweise darauf, dass sich ein sehr früher Besuch (in manchen Untersuchungen in den USA in den ersten sechs Lebensmonaten) und ein hoher Betreuungsumfang in frühpädagogischen Einrichtungen oder der Tagespflege leicht negativ auf soziale Kompetenzen auswirken und tendenziell das Ausmaß an Verhaltensauffälligkeiten steigern. Hingegen wirkt sich die Qualität der besuchten Kindertageseinrichtungen - bei dem gegenwärtig gegebenen Qualitätsniveau - in den ersten Lebensjahren noch nicht auf die sozial-emotionalen Fähigkeiten der Kinder aus. Vielmehr zeigt die differenzierte Längsschnittuntersuchung von Vandell (2011), dass eine hohe Qualität der frühen außerfamilialen Betreuungserfahrungen mit einer Reduzierung von problematischen Verhaltensweisen im Jugendalter einhergeht. Dagegen findet sich bei Kindern ab etwa dem dritten Lebensjahr bis zum Schulbeginn - hier handelt es sich in der Regel um 
institutionelle Betreuungen - keine Beziehung zwischen der Betreuungsdauer und den Sozialkompetenzen der Kinder. Bei diesen Kindern hat aber eine gute Qualität der pädagogischen Prozesse in den Einrichtungen kurz- und längerfristige positive Auswirkungen auf verschiedene Aspekte des Sozialverhaltens.

Gelegentlich finden sich in der Forschung Hinweise darauf, dass ein hoher Zeitumfang institutioneller Betreuung zu einem leicht erhöhten Ausmaß an Verhaltensproblemen führt. Es ist aber wichtig festzuhalten, dass zum einen von diesen erhöhten Verhaltensproblemen nur eine kleine Gruppe von Kindern betroffen ist und zum anderen diese Verhaltensprobleme nicht in einem klinischen Bereich liegen.

Auswirkungen auf kognitiv-leistungsbezogene Kompetenzen von Kindern: Bei den unter dreijährigen Kindern hat der Betreuungsumfang im Allgemeinen kaum Auswirkungen auf die kognitiv-leistungsbezogene Entwicklung, während in fast allen Untersuchungen eine höhere Qualität der frühpädagogischen Einrichtungen kurz- und längerfristig mit besseren Werten im kognitiv-leistungsbezogenen Bereich einhergeht. Dabei sind vor allem die sprachliche Umwelt und die Sprachförderung von Bedeutung. Bei den Kindern ab etwa drei Jahren sind sowohl die Qualität der Einrichtung als auch der Betreuungsumfang von Bedeutung, sodass eine gute Qualität stets mit besseren Werten im kognitiv-leistungsbezogenen Bereich verbunden ist.

Die Effekte der Qualität gehen sowohl von der Prozessqualität, d.h. der Qualität der Anregungen innerhalb einer Einrichtung, als auch von der Strukturqualität, d.h. von den prinzipiell politisch veränderbaren Rahmenbedingungen wie z.B. Gruppengröße, Erzieher-Kind-Relation, Ausbildungsniveau des Fachpersonals, aus. Hier weisen verschiedene Untersuchungen auf positive Auswirkungen von kleineren Gruppen, von einer günstigeren Erzieher-Kind-Relation und von einem höheren - in den Untersuchungen oftmals akademischen - Ausbildungsniveau des Fachpersonals hin. Am deutlichsten zeigen sich die positiven Auswirkungen bei einem höheren Ausbildungsniveau des Personals. Die bisher genannten Untersuchungen analysieren - im Unterschied zu Evaluationen von Interventions-/Modellprogrammen - die Auswirkungen der Qualität der in dem jeweiligen Untersuchungsland üblicherweise vorzufindenden Einrichtungen, die sich in Deutschland gegenwärtig leider eher nur im Bereich der gehobenen Mittelmäßigkeit bewegt (vgl. z.B. Kuger/Kluczniok 2008). Dies ist insofern bedenklich, als es Hinweise darauf gibt, dass längerfristige positive Auswirkungen nur von einer hohen Qualität der frühpädagogischen Einrichtungen ausgehen. Ein Untersuchungsbeispiel hierfür ist das englische „Effective Pre-School and Primary School Education (EPPE 3-11) Project": Die groß angelegte Längsschnittstudie zeigt, dass bis zu einem Alter von zehn Jahren langfristige positive Auswirkungen auf spätere Schulleistungen nur von einer hohen Qualität der frühpädagogischen Einrichtungen hervorgerufen werden (vgl. Sammons et al. 2008). Das bedeutet: Lediglich der Besuch einer frühpädagogischen Einrichtung oder der Besuch einer Einrichtung mit einer niedrigen bis mittleren Qualität lassen keine längerfristigen positiven Auswirkungen wie oben beschrieben erwarten.

Als ein weiteres Beispiel für die positiven Effekte einer qualitativ hochwertigen Erziehung, Bildung und Betreuung vor Schulbeginn können die Ergebnisse des Modellversuchs „Kindergarten der Zukunft in Bayern - KiDZ“ dienen (vgl. Roßbach/Sechtig/ Freund 2010). Der Modellversuch KiDZ war ein Gemeinschaftsprojekt des Bayerischen Staatsministeriums für Unterricht und Kultus, des Bayerischen Staatsministeriums für Ar- 
beit und Sozialordnung, Familie und Frauen, der vbw - Vereinigung der Bayerischen Wirtschaft e.V., unterstützt durch den vbm - Verband der Bayerischen Metall- und Elektroindustrie e.V., sowie der Stiftung Bildungspakt Bayern. Der Modellversuch legte u.a. besonderen Wert auf eine Förderung in den Bereichen frühe Literacy, Mathematik und Naturwissenschaften, indem die Situationen im Kindergartenalltag zur Förderung der Kinder genutzt wurden - auch unter Einschluss von Grundschullehrkräften mit ihren spezifischen, durch eine Hochschulausbildung erworbenen Kompetenzen. Die empirische Evaluation ergab, dass die Teilnahme an KiDZ zu Vorteilen in der kindlichen Entwicklung bzw. im kindlichen Entwicklungsstand führt. Diese Vorteile zeigen sich besonders in mathematischen sowie in sprachlichen und auf den Schriftspracherwerb (Literacy) bezogenen Kompetenzen (z.B. Wissen über Schrift, Buchstabenkenntnisse, erreichte Stufe der Schriftsprachentwicklung, Fähigkeiten in Sprache, Zuhören und Erzählen). Gleichzeitig konnten die Befürchtungen, das stärker kognitiv und schulvorbereitend orientierte KiDZ-Programm könne zu Einbußen in Alltagsfähigkeiten und im Sozialverhalten, zu einem verstärkten kindlichen Problemverhalten, zu einer Reduktion des Wohlbefindens und der Lernfreude sowie zu einer Steigerung der Besorgtheit führen, keinesfalls bestätigt werden. Im Gegenteil: Im Vergleich zu den Kindern der Kontrollgruppe gibt es Hinweise auf eine Reduktion von emotional instabilen Verhaltensweisen und auf eine Erhöhung des Wohlbefindens und der Lernfreude.

Ausgleichende (kompensatorische) Wirkungen für Kinder aus benachteiligten Familien: Die Erwartungen an frühpädagogische Einrichtungen, zu einem Ausgleich sozial bedingter Ungleichheiten beizutragen, sind heute hoch. Die Forschungslage ist allerdings noch unzureichend. In den 1970er Jahren erfolgte z.B. in Norwegen eine Reform des frühkindlichen Betreuungssystems, die zu einer deutlichen Ausweitung der Nutzung von öffentlich unterstützten frühpädagogischen Einrichtungen führte. Diese Ausweitung des Früherziehungssystems insgesamt geht im frühen Erwachsenenalter mit einer verbesserten Schulausbildung, mehr Erfolg auf dem Arbeitsmarkt und einer reduzierten Abhängigkeit vom Sozialstaat einher (vgl. Havnes/Mogstad 2011). Dabei zeigt sich, dass benachteiligte Kinder (insbesondere Kinder von Müttern mit niedrigem Bildungsniveau) am meisten von der frühen Kinderbetreuung profitieren.

Den kompensatorischen Wirkungen für Kinder aus benachteiligten Familien wurde besonders in aufwändigen und intensiven Interventionsprogrammen in den USA nachgegangen. Hier zeigen sich sehr deutlich langfristige positive Effekte auf die Entwicklung der Kinder in den verschiedensten Entwicklungsbereichen (vgl. Blok et al. 2005; Barnett 2008; Leseman 2009; Camilli et al. 2010). Ein schon seit Jahren vielzitiertes Beispiel ist das „Perry Preschool Project“, das in den 1960er Jahren in Ypsilanti (USA) durchgeführt wurde und durch die Reanalysen des Nobelpreisträgers James Heckman (vgl. Heckman et al. 2010) noch einmal an Bekanntheit gewann. Das Projekt gliederte sich in die folgenden Bestandteile: Die Erzieher-Kind-Relation betrug 1:5. Das Erziehungspersonal verfügte in der Regel über eine akademische Ausbildung und besuchte jedes Kind an einem Nachmittag in der Woche für etwa 1,5 Stunden zu Hause. Besonderer Wert wurde auf Elterntreffen und Elternabende gelegt. Das Projekt verbindet somit eine institutionelle Förderung mit einer ausgeprägten familienunterstützenden Komponente. An dem Projekt nahmen 123 drei- und vierjährige Kinder afro-amerikanischer Herkunft mit relativ niedrigen Intelligenzwerten (IQ von 80) aus sehr benachteiligten Sozialschichten teil, die bis zu ihrem 40. Lebensjahr in einer Längsschnittstudie begleitet wurden. Die Kinder in der Inter- 
ventionsgruppe hatten - im Vergleich zu den Kindern in der Kontrollgruppe - eine verbesserte Schullaufbahn und weniger Sonderschulzuweisungen, benötigten weniger Sozialhilfe in späteren Jahren, verfügten über ein höheres durchschnittliches Jahreseinkommen und zeigten seltener auffälliges Verhalten (z.B. reduzierte Kriminalität). Auch für den Steuerzahler zahlen sich Bildungsinvestitionen dieser Art aus, erhält er doch für jeden US-Dollar Investition in die Frühförderung später im Lebensverlauf zwölf US-Dollar zurück (vgl. Schweinhart et al. 2005; die Schätzungen von Heckman et al. 2010 liegen mit einer „rate of return“ zwischen sieben und zehn Prozent etwas niedriger).

Trotz dieser eindrucksvollen langfristigen Wirkungen auf den Lebensverlauf ist zu berücksichtigen, dass das Perry Preschool Project eine sehr aufwändige und qualitativ hochwertige Intervention darstellt. $\mathrm{Ob}$ es sich bei den positiven Langzeitauswirkungen um kompensatorische Effekte im engeren Sinne handelt, die die Schere zwischen benachteiligten und bevorzugten Kindern verringert oder sogar schließt, kann mithilfe des Perry Preschool Project allerdings nicht beantwortet werden, da nur Kinder aus benachteiligten Familien teilnahmen. Möglicherweise zeigen sich bei nicht benachteiligten Kindern Effekte in der gleichen Richtung. Wird nicht die Teilnahme an einem besonderen Interventionsprogramm, sondern der Besuch einer üblicherweise vorzufindenden Einrichtung mit der dort gegebenen Qualität betrachtet, so zeigen sich ebenfalls positive Auswirkungen auf die Entwicklung von Kindern aus benachteiligten Familien. Unklar ist aber der Forschungsstand mit Blick auf die Frage, ob sich für Kinder in diesen Einrichtungen besondere Effekte zeigen, die sozial bedingte Nachteile auszugleichen helfen. Solche kompensatorischen Effekte finden sich nicht durchgängig. Allerdings gibt es in verschiedenen Studien Hinweise, dass eine hohe Qualität der Förderung in einer frühpädagogischen Einrichtung durchaus familiale Benachteiligungen bzw. solche, die durch individuelle Risikolagen der Kinder entstehen, reduzieren kann (vgl. Sammons et al. 2008; Dearing/McCartney/Taylor 2009; Hall et al. 2009; Burchinal et al. 2010). Festgehalten werden kann damit: Der Besuch einer üblicherweise vorzufindenden frühpädagogischen Einrichtung und ihre Qualität wirken sich positiv auf die Entwicklung von Kindern aus benachteiligten Familien aus. Um besondere Fördereffekte für benachteiligte Kinder zu erreichen, werden vermutlich entweder aufwändige Interventionen oder zumindest eine hohe pädagogische Qualiät in den frühpädagogischen Einrichtungen und eine begleitende Familienarbeit benötigt.

Auswirkungen auf die Familien der Kinder. Frühpädagogische Einrichtungen sollen zudem einen Beitrag zur Vereinbarkeit von Familien- und Erwerbstätigkeit leisten, was bei der heute gemeinhin noch vorherrschenden geschlechtsspezifischen Arbeitsteilung speziell für die Mütter gilt. Ganz allgemein wird dies zunächst durch das quantitative Angebot an Betreuungsplätzen erreicht, das zu den Bedürfnissen der Eltern passt. Allerdings ist letztlich nicht allein das quantitative Angebot ausschlaggebend. In verschiedenen Untersuchungen wurden positive Auswirkungen der Qualität der frühpädagogischen Einrichtungen auf die Erwerbstätigkeit der Mütter festgehalten (vgl. z.B. Mason/Duberstein 1992; Vandell/Wolfe 2000; zusammenfassend Roßbach 2005). Tendenziell fördert eine hohe Qualität der Einrichtung - im Vergleich zu einer niedrigen Qualität - die (Wieder-) Aufnahme einer Erwerbstätigkeit, ihre Stabilität und ihren zeitlichen Umfang. Insbesondere führt sie zu weniger Fehltagen und mehr Pünktlichkeit am Arbeitsplatz und trägt möglicherweise zu einer besseren Arbeitsproduktivität bei. Eine gute Qualität der frühpädagogischen Einrichtung erhöht somit die mütterliche bzw. elterliche Zufriedenheit mit 
der Betreuung der Kinder und fördert darüber die Bereitschaft zur Teilnahme am Arbeitsmarkt. Eine Erklärung für diese Effekte liefert die These, dass Mütter insgesamt beruhigter sind, wenn sie wissen, dass während der Zeit ihrer Erwerbstätigkeit in der Kinderbetreuungseinrichtung das Beste für ihre Kinder getan wird und sie pädagogisch gut betreut werden (vgl. Vandell/Wolfe 2000).

Zusammenfassend lässt sich festhalten, dass der Besuch einer frühpädagogischen Einrichtung und ihre Qualität förderlich für die Entwicklung der Kinder im sozial-emotionalen wie im kognitiv-leistungsbezogenen Bereich sind und dass längerfristige positive Effekte vor allem von einer hohen Förderqualität ausgehen. Weiterhin ist eine hohe Qualität - entweder in den üblicherweise vorzufindenden Einrichtungen oder in Interventionsprogrammen - wichtig, damit besondere Effekte für Kinder aus benachteiligten Familien erzielt werden, d.h., damit individuelle oder familial bedingte Ungleichheiten zumindest ansatzweise reduziert werden. Schließlich wirkt sich eine gute Qualität nicht nur auf die Kinder, sondern auch auf die Familien aus. Dabei ist jedoch zu berücksichtigen, dass die pädagogische Förderqualität in deutschen frühpädagogischen Einrichtungen gegenwärtig häufig nicht besonders hoch ist. Verschiedene Untersuchungen, die die Förderqualität über systematische Beobachtungsverfahren analysiert haben, verweisen auf eine im Durchschnitt mittelmäßige Qualität (vgl. Tietze/Roßbach/Grenner 2005; Kuger/Kluczniok 2008). Es lohnt sich also, die heutige Qualität der frühpädagogischen Einrichtungen zu steigern und in höhere Qualität zu investieren. Neben Verbesserungen der unmittelbaren Förderprozesse in den Einrichtungen sind vor allem Verbesserungen in den Strukturmerkmalen von Kindertageseinrichtungen bedeutsam. Zentrale Ansatzpunkte sind in diesem Zusammenhang die Verkleinerung von Gruppen, die Reduzierung der Anzahl von betreuten Kindern pro Erzieherin bzw. Erzieher sowie Verbesserungen der Aus-, Fort- und Weiterbildung des pädagogischen Fachpersonals. Die Untersuchungsergebnisse weisen wiederholt auf die besondere Bedeutung des Qualifikationsniveaus des Fachpersonals hin.

\section{Rolle des Qualifikationsniveaus und der Kompetenzen der pädagogischen Fachkräfte}

Etwa 72 Prozent des pädagogischen Fachpersonals in Kindertageseinrichtungen in Deutschland bestand im Jahr 2010 aus Erzieherinnen und Erziehern, die an Fachschulen bzw. Fachakademien für Sozialpädagogik ausgebildet wurden; gefolgt von an Berufsfachschulen ausgebildeten Kinderpflegerinnen bzw. Kinderpflegern (14 Prozent). Nur ein sehr geringer Teil des pädagogischen Fachpersonals (3,5 Prozent) kann auf eine akademische Ausbildung verweisen. Wie bereits aufgezeigt, spielt das Ausbildungsniveau des Fachpersonals eine wichtige Rolle, wenn über positive Auswirkungen des Besuchs einer frühpädagogischen Einrichtung berichtet wird. Allerdings wird in vielen Untersuchungen zum einen das Ausbildungsniveau nur sehr pauschal betrachtet; zum anderen gibt es gegenwärtig (noch) keine adäquate Untersuchung in Deutschland, die Aufschluss darüber gibt, wie sich Unterschiede im Ausbildungsniveau des Fachpersonals (z.B. fachschulische versus hochschulische Ausbildung) auf die Gestaltung der pädagogischen Praxis und die Entwicklung der Kinder auswirken. Deshalb muss hier auf Untersuchungen aus anderen Staaten zurückgegriffen werden. Aufgrund von unterschiedlichen Rahmenbedingungen 
und unterschiedlichen Ausbildungssystemen sind diese Forschungsergebnisse nicht immer direkt auf die deutsche Situation übertragbar.

Bedeutung des formalen Bildungsabschlusses. Im „Effective Provision of Pre-School Education (EPPE) Project" in England zeigt sich ein positiver Zusammenhang zwischen dem Qualifikationsgrad der Mitarbeiterinnen und Mitarbeiter und der Einrichtungsqualität (vgl. Taggart et al. 2000; Sylva et al. 2004). Je höher die Qualifikation des Personals, insbesondere der Einrichtungsleitungen, ist, desto höher ist die beobachtete Förderqualität in den Einrichtungen und desto größere Entwicklungsfortschritte machen die Kinder: „Der Qualifikationsgrad des Personals erweist sich als wichtigster Einflussfaktor für die Qualität der Einrichtungen und steht in engem Zusammenhang mit den Entwicklungsfortschritten der Kinder speziell in den Bereichen der sozialen Entwicklung und den Vorläuferfähigkeiten des Lesens" (Sylva et al. 2004: 159). Aus dem EPPE-Projekt geht weiterhin hervor, dass Fachkräfte mit einem Universitätsabschluss Kinder eher zu nachhaltigem Denken und zu kognitiv anspruchsvollen Aktivitäten anregen als weniger qualifiziertes Personal. Besonders bedeutsam ist ein Effekt der Teamzusammensetzung: Geringer qualifizierte Betreuungskräfte profitieren in Bezug auf ihre pädagogische Arbeit, wenn sie mit qualifizierteren Kolleginnen und Kollegen zusammenarbeiten. Gelegentlich wird befürchtet, dass ein höheres formales Ausbildungsniveau (z.B. ein Bachelorabschluss an einer Hochschule) zu einer weniger effektiven Interaktion des Fachpersonals mit den Kindern führen könne. Die Ergebnisse von zwei groß angelegten Untersuchungen in den USA verweisen aber auf das Gegenteil: Fachkräfte, die einen Bachelorabschluss mit einer Spezialisierung auf Frühpädagogik besitzen, zeigen ein höheres Ausmaß an Sensitivität und Responsivität im Umgang mit Kindern als Fachkräfte mit einem niedrigeren Ausbildungsabschluss (vgl. Howes 1997).

Auf der Basis eines älteren Literaturüberblicks folgert Whitebook (2003) für die USA, dass die empirische Forschungslage für eine Hochschulausbildung spricht. Es handelt sich dabei um einen vierjährigen Bachelorstudiengang auf Collegeniveau mit einer Spezialisierung in Fragen der Frühpädagogik oder Entwicklungspsychologie. Allerdings betont Whitebook, dass aus der prinzipiellen Bedeutung eines Hochschulabschlusses nicht zwingend folgt, dass eine solche Ausbildung für alle Fachkräfte eines Teams erforderlich ist. Für eine Hochschulausbildung sprechen aber ebenfalls die Ergebnisse einer Metaanalyse von 32 Untersuchungen von Kelley und Camilli (2007). Sie finden einen positiven Effekt eines Bachelorabschlusses gegenüber einem niedrigeren Ausbildungsniveau im Hinblick auf verschiedene Maße der Förderqualität und der kindlichen Entwicklung.

Weniger deutlich sind dagegen die Ergebnisse einer Untersuchung von Early et al. (2006; vgl. auch Oberhuemer/Schreyer 2010: 487f.). Hier hatte das formale Ausbildungsniveau des Fachpersonals (Bachelorabschluss oder höher versus einen nicht akademischen Abschluss) keinen Einfluss auf die in den untersuchten frühpädagogischen Gruppen realisierte Förderqualität. Zwar übten die höheren Abschlüsse einen positiven Einfluss auf die Entwicklung der Kinder in mathematischen Kompetenzen aus, nicht aber auf die sprachlichen Kompetenzen. Ebenfalls geringe Effekte stellen Early et al. (2007) bei einem systematischen Vergleich von sieben größeren empirischen Studien fest. Bei der Bewertung der Forschungslage muss jedoch berücksichtigt werden, dass die meisten Untersuchungen lediglich pauschal den Ausbildungsabschluss erheben, ohne genauere Merkmale und Inhalte der Hochschulausbildungen zu betrachten. Dafür, dass sich keine oder nur geringe Effekte des formalen Ausbildungsniveaus zeigen, formulieren z.B. Early et al. (2007: 573ff.) drei 
mögliche Erklärungen, die auch für die Situation in Deutschland relevant sind: Die Hochschulausbildung als solche könnte die Studierenden nicht adäquat auf ihre zukünftigen Aufgaben vorbereitet haben, indem die Curricula z.B. zu wenig Rücksicht auf neueres Forschungswissen nähmen oder zu wenig Wert auf die Bedeutung einer respektvollen Beziehung zwischen den Kindern und den Fachkräften als Grundlage für kognitives Lernen legten. Damit wird die Frage nach einem adäquaten Ausbildungscurriculum an den Hochschulen aufgeworfen. Das formale Ausbildungsniveau wird dementsprechend nur als eine notwendige, aber nicht als eine hinreichende Bedingung zur Verbesserung der Praxis angesehen. Es kommt auch auf das Curriculum und die Ausbildungsqualität an. Das hochschulisch ausgebildete Personal könnte in der Praxis nicht genügend Unterstützung erfahren haben, um das in der Hochschulausbildung Gelernte auch erfolgreich umzusetzen. Dies weist auf die Bedeutung einer unterstützten Einmündungsphase hin, besonders dann, wenn nur geringe Praxisanteile in das Studium integriert werden. Insgesamt ist damit auch die Frage nach der systematischen Verknüpfung von theoretischen Ausbildungsinhalten an den Hochschulen - aber auch an den Fachschulen - und dem Lernort Praxis aufgeworfen.

Eine weitere Erklärung könnten Selektionseffekte bei den untersuchten frühpädagogischen Einrichtungen liefern, die finanziell durch die öffentliche Hand unterstützt werden (state-funded pre-kindergarten): Gegenüber dem privaten Bereich werden hier bessere Gehälter bezahlt, die möglicherweise die besten Fachkräfte ohne Bachelorabschluss anziehen. Andererseits liegen die Gehälter im Grundschulbereich höher, sodass die besten Absolventinnen und Absolventen mit einem Bachelorabschluss vermehrt in diesen Bereich abwandern. Als Ergebnis würden dadurch Qualitätsunterschiede des pädagogischen Personals nivelliert, die auf unterschiedliche Ausbildungsebenen zurückgeführt werden könnten. Damit stellt sich die Frage, wer mit welchem Ausbildungsniveau unter den gegebenen Umständen (speziell im Hinblick auf die Bezahlung) in das frühpädagogische Feld eintritt und darin verbleibt. Systematische Untersuchungen hierzu liegen in Deutschland noch nicht vor. Informelle Berichte von Einzelfällen zeigen aber, dass auch in Deutschland Absolventinnen und Absolventen der Bachelorstudiengänge für Frühpädagogik oftmals in andere Felder abwandern (z.B. Grundschullehramt studieren).

Bedeutung spezifischer Kompetenzen des Fachpersonals: Derzeit liegen wenige Untersuchungen dazu vor, welche Kompetenzen des Fachpersonals sich wie auf die pädagogische Arbeit und die Entwicklung der Kinder auswirken. Systematische und umfangreiche Fallstudien im Rahmen der groß angelegten Längsschnittuntersuchung in England, des EPPEProjekts (vgl. Siraj-Blatchford et al. 2002; Sylva et al. 2004), verweisen auf die besondere Bedeutung von fünf Bereichen einer effektiven und entwicklungsfördernden Arbeit mit Kindern im Alter von drei bis fünf Jahren: die Qualität der verbalen Erwachsenen-KindInteraktion, das didaktische bzw. curriculare Fachwissen der Erzieherinnen und Erzieher, das Fachwissen über kindliche Lernprozesse, die Fähigkeit der Erwachsenen, Kinder bei Konfliktlösungen zu unterstützen, sowie Hilfen für Eltern, um die kognitive Entwicklung der Kinder zu Hause zu fördern. Besonders Fachwissen in verschiedenen Entwicklungsbereichen und fachdidaktisches Wissen verweisen auf Wissenskomponenten, die schon seit längerem in der Lehrerbildungsforschung thematisiert werden (vgl. z.B. Baumert/ Kunter 2006) und die in der Regel durch ein Hochschulstudium erworben werden.

Auswirkungen von Fort- und Weiterbildung: $\mathrm{Da}$ in Deutschland zurzeit nur ein geringer Anteil des Personals auf Hochschulebene ausgebildet wird, ist die Frage der Fort- und 
Weiterbildung des Personals von besonderer Bedeutung. Wirkungsstudien hierzu gibt es nur wenige. Ohne Anspruch auf Vollständigkeit wird auf zwei Untersuchungen verwiesen: In einer Metaanalyse von 17 Studien kommen Fukkink/Lont (2007) zu dem Ergebnis, dass spezifische, auf die Adressaten abgestimmte Fortbildungsveranstaltungen (specialized trainings) positive Auswirkungen auf die Kompetenzen des frühpädagogischen Personals haben. Ebenfalls zeigen sich Effekte auf den kindlichen Entwicklungsstand, die allerdings nicht signifikant sind. Die Auswirkungen auf die Kompetenzen des Personals fallen im Bereich der Fertigkeiten etwas niedriger aus als in den Bereichen Wissen und Orientierungen. Auswirkungen auf pädagogische Orientierungen finden sich auch in einer spezifischen Fortbildung, dem Child Development Associate Training. Diese richtet sich speziell an jene Fachkräfte, die entweder keinen Collegeabschluss besitzen oder während des Studiums am College keine frühpädagogischen Inhalte vermittelt bekommen haben (vgl. Heisner/Lederberg 2011). Aus diesen Ergebnissen lässt sich schließen, dass sich systematische und gezielte Fort- und Weiterbildung lohnt.

Auch wenn der derzeitige Forschungsstand zum Qualifikationsniveau und zu den Kompetenzen des Personals frühpädagogischer Einrichtungen in einigen Aspekten noch lückenhaft ist, lässt sich zusammenfassend festhalten: Gute Qualifikationen des pädagogischen Fachpersonals und vor allem auch eine akademische Hochschulausbildung sind bedeutsam. Das bedeutet jedoch nicht, dass das gesamte Personal einer Einrichtung akademisch ausgebildet sein muss. Es liegt nahe, dass insbesondere die akademische Ausbildung der Einrichtungsleitungen für die Weiterentwicklung der Einrichtungen eine wichtige Rolle spielt. Zudem sind Teameffekte zu erwarten, indem sich die pädagogische Arbeit von niedriger qualifizierten Fachkräften durch den Einfluss von höher qualifizierten in einer Einrichtung verbessert. Ebenso ist das konkrete Ausbildungscurriculum an den Hochschulen - wie auch an den Fachschulen - von Bedeutung. Die gefundene Relevanz von Fachwissen und fachdidaktischem Wissen legt es nahe, sich in der fachschulischen wie auch der hochschulischen Ausbildung stärker an Kompetenzstrukturmodellen zu orientieren, wie sie aus der Lehrerbildungsforschung bekannt sind. Für eine effektive Umsetzung des in der Ausbildung Gelernten scheinen auch eine gezielte Unterstützung bei der Berufseinmündung und eine systematische Verknüpfung der theoretischen Lerninhalte mit dem Lernort Praxis wichtig. Schließlich zahlt sich die gezielte und systematische Fort- und Weiterbildung des Personals aus. Insgesamt lohnt es sich somit, in eine qualitativ hochwertige Aus-, Fort- und Weiterbildung des Personals in frühpädagogischen Einrichtungen zu investieren.

\section{Gegenwärtige Ausbildungssituation und Handlungsbedarf für die nächsten Jahre}

Der Bereich der Kindertageseinrichtungen umschließt Krippen, Kindergärten, altersgemischte Einrichtungen sowie Horte für die außerschulische und außerunterrichtliche Betreuung von Kindern im Schulalter und gehört rechtlich und organisatorisch zum Jugendhilfebereich (vgl. Roßbach 2008). Von allen Kindertageseinrichtungen waren im Jahr 2011 ein Drittel in öffentlicher und zwei Drittel in freier Trägerschaft (vgl. Statistisches Bundesamt 2011). 2,9 Prozent aller Einrichtungen betreuen ausschließlich Kinder unter drei Jahren, 43 Prozent nehmen Kinder von zwei bis acht Jahren auf, und 6,9 Prozent der 
Einrichtungen bieten die außerschulische Betreuung von Kindern im Schulalter von fünf bis 14 Jahren an. Den größten Anteil mit 47,2 Prozent nehmen solche Einrichtungen ein, die Kinder aller Altersgruppen betreuen. Kindertagesstätten sind mit durchschnittlich jeweils 3,3 Gruppen in der Regel eher kleinere Einrichtungen; der Anteil der Einrichtungen mit mehr als fünf Gruppen beträgt lediglich 10,6 Prozent. Durchschnittlich sind umgerechnet in Vollzeitstellen 6,9 Personen - pädagogisches Fachpersonal, Leitungspersonal in einer Einrichtung beschäftigt, wobei das Leitungspersonal in der Regel auch in den Gruppen mitarbeitet. In lediglich 38,4 Prozent der Einrichtungen beschränkt sich der Arbeitsbereich dieses Personals auf die Leitungstätigkeit.

Gegenwärtige Struktur des frühpädagogischen Fachpersonals: Im Jahr 2010 waren 379.006 pädagogische Fachkräfte in deutschen Kindertageseinrichtungen - ungeachtet der Angestellten in Horten - beschäftigt. Dies entspricht 305.388 Vollzeitäquivalenten. Damit stieg die Anzahl pädagogischer Fachkräfte im Zeitraum von 2002 bis 2010 um rund 78.000 Personen, d.h. um knapp 59.000 Vollzeitäquivalente (vgl. Aktionsrat Bildung 2012 auf der Basis von Autorengruppe Bildungsberichterstattung 2010). Die größte Gruppe bilden die an Fachschulen bzw. Fachakademien ausgebildeten Erzieherinnen und Erzieher (71,7 Prozent im Jahr 2010), gefolgt von den an Berufsfachschulen ausgebildeten Kinderpflegerinnen und Kinderpflegern (14,4 Prozent). Hinzu kommen an Fachhochschulen oder Universitäten ausgebildete Diplom-Sozialpädagoginnen und -Sozialpädagogen sowie Diplom-Pädagoginnen und -Pädagogen u.a. (2,9 Prozent) sowie Absolventinnen und Absolventen sonstiger Sozial- und Erziehungsberufe (1,2 Prozent), Gesundheitsberufe und Berufe mit anderen Abschlüssen (3,7 Prozent) sowie Praktikantinnen und Praktikanten (2,4 Prozent) und Personen ohne Ausbildung (3,7 Prozent) (vgl. Aktionsrat Bildung 2012, Tabelle 3 im Anhang des Gutachtens). Trotz intensiver Diskussion um eine Akademisierung der Ausbildung stieg der Anteil des Personals mit einer Hochschulausbildung in den letzten Jahren nur geringfügig an - von 2,2 Prozent im Jahr 2002 auf 3,5 Prozent im Jahr 2010. Die Akademisierung des Personals in den Kindertageseinrichtungen bezog sich dabei vor allem auf das Leitungspersonal, wobei große Unterschiede zwischen den Bundesländern festzustellen sind (vgl. Fuchs-Rechlin 2009).

Der niedrige Akademisierungsgrad darf aber nicht darüber hinwegtäuschen, dass im Jahr 2010 71,7 Prozent des Personals über eine fachlich einschlägige, an Fachschulen für Sozialpädagogik absolvierte, Erzieherinnen- bzw. Erzieherausbildung verfügen. Mithin können drei Viertel des pädagogischen Personals in deutschen Kindertageseinrichtungen eine akademische oder eine mindestens dreijährige einschlägige Fachschulausbildung vorweisen. Ein solch hoher Anteil formal-fachlicher Qualifikation findet sich weder in den USA noch in den meisten europäischen Ländern (vgl. Thole 2010).

Für die gegenwärtige Diskussion um eine Professionalisierung des frühpädagogischen Personals sind Erzieherinnen und Erzieher sowie Absolventinnen und Absolventen der frühpädagogischen Bachelorstudiengänge von besonderer Bedeutung. Die Sonderauswertung des Mikrozensus 2008 zur Situation der Erzieherinnen und Erzieher in Deutschland von Fuchs-Rechlin (2010) zeichnet folgendes Bild: 97,7 Prozent des im Mikrozensus erfassten Erziehungspersonals ist weiblich, 7,6 Prozent der Fachkräfte haben einen Migrationshintergrund, und über die Hälfte (54,4 Prozent) hat eigene Kinder. Sieben Prozent verfügen über einen Hauptschulabschluss, und fast zwei Drittel (63,5 Prozent) haben die mittlere Reife absolviert. Darüber hinaus besitzt mit 30 Prozent ein relativ hoher Anteil 
der Erzieherinnen und Erzieher die Hochschulreife (16,0 Prozent Fachhochschulreife und 13,6 Prozent allgemeine oder fachgebundene Hochschulreife), und damit die formale Qualifikation für ein Studium. Von allen Erzieherinnen sind nur 49,6 Prozent vollzeitbeschäftigt; hingegen arbeiten 72,3 Prozent des männlichen Erziehungspersonals in Vollzeit. Damit liegt die Vollzeitquote der Erzieherinnen im Vergleich zu anderen Berufsgruppen niedriger. Insgesamt sind 14,5 Prozent der Erzieherinnen und Erzieher (hier zusammen mit Kinderpflegerinnen und Kinderpflegern) befristet angestellt; über alle Berufe hinweg liegt die Befristungsquote nur bei acht Prozent. Allerdings steigt die Quote auf 51,4 Prozent bei dem unter 25-jährigen Erziehungspersonal. In diesem Zusammenhang wird häufig auf eine kritische Berufseinmündungsphase geschlossen, die möglicherweise bei einem nicht stabilen Beschäftigungsverhältnis eine Abwanderung aus dem frühpädagogischen Feld befördern kann. 65,3 Prozent der Fachkräfte mit einer frühpädagogischen Ausbildung verbleiben im Feld der frühpädagogischen Einrichtungen. Abwanderungen nehmen mit zunehmendem Lebensalter zu und fallen bei Männern und Erzieherinnen mit Migrationshintergrund höher aus.

Fuchs-Rechlin (2010: 38ff.) hat darüber hinaus versucht, näherungsweise das Nettoeinkommen von Erzieherinnen und Erziehern sowie Kinderpflegerinnen und Kinderpflegern im Berufsgruppenvergleich zu schätzen (siehe Aktionsrat Bildung 2012, Tabelle 5 im Anhang des Gutachtens). Auf der Basis des Mikrozensus 2008 ergibt sich für vollzeitbeschäftigte Erzieherinnen ein Nettoeinkommen von 1.365 Euro pro Monat. Dieses liegt etwa auf dem Niveau des Nettoeinkommens aller weiblichen Beschäftigten in Höhe von 1.387 Euro. Fuchs-Rechlin (2010: 3) folgert: „Entwarnung bei der ökonomischen Situation: ErzieherInnen und KinderpflegerInnen können bei einer Vollzeitbeschäftigung ihren Lebensunterhalt mehrheitlich aus eigener Erwerbsarbeit bestreiten." Allerdings liegt ihr Bruttomonatsverdienst nach der Verdienststrukturerhebung 2006 des Statistischen Bundesamts (2009; siehe auch Aktionsrat Bildung 2012) im Vergleich zu anderen pädagogischen Berufen eher am unteren Ende: So beträgt der Bruttomonatsverdienst von vollzeitbeschäftigten Kindergärtnerinnen und Kinderpflegerinnen 2.514 Euro, während Realschul-, Volksschul- und Sonderschullehrerinnen auf 3.233 Euro und Gymnasiallehrerinnen auf 3.573 Euro kommen.

In einer nicht repräsentativen Befragung des weiblichen Erziehungspersonals durch die Gewerkschaft Erziehung und Wissenschaft (GEW), bei der insgesamt 1.900 Fragebögen ausgewertet werden konnten, wurde u.a. die hohe Weiterbildungsmotivation der befragten Erzieherinnen deutlich. Im Hinblick auf die Akzeptanz einer berufsbegleitenden Hochschulausbildung (vgl. Forschungsverbund Deutsches Jugendinstitut und Universität Dortmund 2007: 17f.) gaben neun Prozent der Befragten an, sie würden einen solchen Studienabschluss auf jeden Fall erwerben; weitere 48 Prozent können sich dies unter Umständen vorstellen (z.B. bei einem nahegelegenen Hochschulstandort, der Möglichkeit eines Fernstudiums, der Anrechnung bisheriger Fortbildungsleistungen). Am höchsten ist die Akzeptanz bei Erzieherinnen unter 30 Jahren. Selbst innerhalb der Altersgruppe der 30- bis 40-Jährigen würden 17 Prozent auf jeden Fall und 62 Prozent unter Umständen ein berufsbegleitendes Hochschulstudium aufnehmen. Damit besteht erhebliches Potenzial für eine berufsbegleitende Hochschulausbildung. Eine Befragung der Leiter von Fachschulen und Berufsfachschulen ergab allerdings, dass sie ihre Absolventinnen und Absolventen zu einem großen Teil (zwei Drittel) für nicht studierfähig halten und insbesondere Schwierigkeiten mit der deutschen Sprache sowie Defizite in Mathematik sehen (vgl. For- 
schungsverbund Deutsches Jugendinstitut und Universität Dortmund 2007; Rudolph 2010).

Wenngleich Studien zu den Absolventinnen und Absolventen der neuen frühpädagogischen Bachelorstudiengänge in Deutschland gegenwärtig erst im Entstehen sind, liegen über die Studierenden in frühpädagogischen Bachelorstudiengängen bereits Informationen aus einer Befragung im Sommersemester 2009 vor (vgl. Helm 2010). Insgesamt konnten schriftliche Fragebögen von 1.000 Studierenden ausgewertet werden. Auch hier sind die Studierenden in der überwiegenden Mehrzahl weiblich (93,1 Prozent). Dies lässt erkennen, dass Männer nicht verstärkt an einer Tätigkeit in frühpädagogischen Einrichtungen interessiert sind. 14,8 Prozent der Studierenden in frühpädagogischen Bachelorstudiengängen haben eigene Kinder - ein Anteil, der im Vergleich zum Bundesdurchschnitt von fünf Prozent der Studierenden als hoch einzustufen ist. Des Weiteren kommen die Studierenden der frühpädagogischen Studiengänge häufiger als in anderen Studienfächern aus nicht akademischen Familien und haben den Zugang zur Hochschule erst im Zuge einer Berufsausbildung erlangt. 59,1 Prozent der Befragten haben die allgemeine Hochschulreife abgelegt, während 31,5 Prozent die Fachhochschulreife und 9,5 Prozent die fachgebundene Hochschulreife vorweisen können. 44,4 Prozent der Studierenden haben bereits vor dem Studium eine Ausbildung abgeschlossen, was deutlich über dem bundesdeutschen Durchschnitt liegt. Der häufigste dieser Abschlüsse war die Ausbildung zur Erzieherin bzw. zum Erzieher, sodass für einen doch recht großen Teil der Studierenden (insgesamt 29 Prozent aller Befragten) fachlich einschlägige berufliche Erfahrungen kennzeichnend sind. Zudem war etwa ein Viertel aller Befragten, die vor Studienbeginn im frühpädagogischen Bereich tätig waren oder während des Studiums noch tätig sind, als Leitungen von Kindertagesstätten beschäftigt. Für Studierende, die bereits vor dem Studium eine sozialpädagogische Qualifikation erworben haben, spielen berufliche Aufstiegschancen eine große Rolle. Systematische und umfangreichere Verbleibstudien der Bachelorabsolventinnen und -absolventen stehen aktuell jedoch noch aus.

Personalbedarf in den nächsten Jahren: Die Frage, inwieweit eine Erhöhung des Anteils an akademisch ausgebildetem Personal kurzfristig möglich ist, wird zum Teil überlagert durch die beschlossene Einführung eines Rechtsanspruchs auf eine Tagesbetreuung für Kinder ab dem vollendeten ersten Lebensjahr ab dem 1. August 2013. Wie bereits erwähnt, müssen dafür im Zeitraum von heute bis August 2013 noch etwa 300.000 Plätze in Kinder- tageseinrichtungen und Kindertagespflege geschaffen werden (vgl. Schilling 2011a). Modellrechnungen im Auftrag der Weiterbildungsinitiative Frühpädagogische Fachkräfte (WiFF) verweisen sowohl auf regionale Personallücken als auch auf Personalüberhänge und sehen keinen flächendeckenden Personalnotstand (vgl. Rauschenbach/Schilling 2010).

Auf der Basis von Schätzungen des Aktionsrats Bildung (2012; vgl. ausführlicher Schilling 2011b) besteht für Deutschland bis zum Jahr 2013 ein Bedarf von etwa 77.000 Vollzeitkräften; für den Zeitraum 2013 bis 2020 werden weitere 119.000 Vollzeitkräfte benötigt werden. Somit beläuft sich für die Zeitspanne von 2010 bis 2020 der Personalbedarf in Kindertageseinrichtungen auf etwa 196.000 Vollzeitkräfte. In den alten Bundesländern wird es dabei bis zum Jahr 2013 einen Fehlbedarf von über 23.000 Personen geben und nach 2013 einen Überschuss an ausgebildetem Personal. Im Gegensatz dazu übersteigt das Fachkräftepotenzial in den neuen Bundesländern im gesamten Zeitraum den Personalbedarf. Insgesamt liegt das Fachkräftepotenzial in Deutschland bis zum Jahr 
2020 um etwa 11.500 Vollzeitstellen höher als der Personalbedarf. Zwar müssen in den alten Bundesländern noch deutliche Anstrengungen unternommen werden, damit beim heutigen Stand des Fachkräftepotenzials quantitativ hinreichend Personal bis zum Jahr 2020 auf den verschiedenen Ebenen ausgebildet wird. Allerdings stellt sich die Situation für Deutschland insgesamt insofern entspannt dar, als zum einen kein „Personalnotstand“ die notwendige Ausweitung der Plätze für unter dreijährige Kinder verhindert und zum anderen auch nicht befürchtet werden muss, dass aufgrund von Personalknappheit kein Spielraum für Reformen der Ausbildung besteht (siehe dazu die quantitativen Abschätzungen des Aktionsrats Bildung 2012 und Schilling 2011b).

Letztlich dürfte aber die Situation in den nächsten Jahren entscheidend von der Attraktivität des frühpädagogischen Bildungs- und Betreuungsbereichs für die Absolventinnen und Absolventen abhängen. Dies wird u.a. von den Verdienstmöglichkeiten in diesem Feld bestimmt. Verschiedene Stellen fordern deshalb eine Überprüfung bzw. Erhöhung der tariflichen Vergütungen im frühpädagogischen Bereich für Absolventinnen und Absolventen sowohl der Fachschulen als auch der Hochschulen (vgl. z.B. Rudolph 2010; Deutscher Verein für öffentliche und private Fürsorge 2011). Um dem kurzfristigen Personalmangel im Bereich der frühpädagogischen Einrichtungen zu begegnen, werden verschiedene Strategien vorgeschlagen (vgl. z.B. Rauschenbach/Schilling 2010; Bundesarbeitsgemeinschaft der Freien Wohlfahrtspflege (BAGFW) 2011; Jugend- und Familienministerkonferenz (JFMK) 2011): Steigerung der Ausbildungskapazitäten an Fach- und Hochschulen; umfassende Personalentwicklungsmaßnahmen zum Verbleib der (älteren) Fachkräfte im Beruf; Erleichterung der Berufseinmündung; Erhöhung der wöchentlichen Arbeitszeit Teilzeitbeschäftigter; Werbemaßnahmen für einen verstärkten Wiedereinstieg in den Beruf; Nutzung des Potenzials arbeitslos gemeldeter Fachkräfte; Nutzung des Ausbildungspotenzials angrenzender Länder und Gewinnung von Fachkräften mit Migrationshintergrund; Qualifizierungsprogramme für Quereinsteiger (inklusive der Gewinnung von mehr männlichem Personal); mehr Werbung für den Beruf durch verschiedene Instanzen und Konzeptualisierung von Praktika sowie des Freiwilligen Sozialen Jahrs in frühpädagogischen Einrichtungen; nicht zuletzt ein flexibler Wechsel des Personals zwischen der Kindertagespflege und den Kindertageseinrichtungen.

Als ein weiteres wichtiges Ergebnis der verschiedenen Modellrechnungen muss festgehalten werden, dass beim gegenwärtigen Stand der einschlägigen Studiengänge an den Hochschulen bis zum Jahr 2020 eine Vollakademisierung des Personals nicht möglich sein wird, da die Kapazitäten der frühpädagogischen Hochschulausbildung heute zu begrenzt sind. Damit wird die Ausbildung des pädagogischen Personals in der frühkindlichen Bildung noch für längere Zeit auf zwei Ebenen stattfinden, an den Fachschulen und an den Hochschulen. Hinzu kommt gegenwärtig noch die Ebene der berufsfachschulischen Ausbildung zur Kinderpflegerin bzw. zum Kinderpfleger und zur Sozialassistentin bzw. zum Sozialassistenten.

Die besondere Herausforderung besteht deshalb in der optimalen Gestaltung der Funktionen und Rollen der Arbeit in Einrichtungsteams mit unterschiedlichen Ausbildungen sowie in der sukzessiven Erhöhung der Qualifikationen. Der Bedarf an akademisch ausgebildetem Personal zeigt sich noch deutlicher, falls bis zum Jahr 2020 in jeder Kindertageseinrichtung eine akademisch ausgebildete Fachkraft (z.B. in der Einrichtungsleitung) beschäftigt würde. Im März 2011 gab es in Deutschland 51.484 Kindertageseinrich- 
tungen (vgl. Statistisches Bundesamt 2011). Im Rahmen des Ausbaus von Betreuungsplätzen für unter dreijährige Kinder dürfte sich an der Zahl der Einrichtungen wenig ändern. Für das Jahr 2020 ist mit etwa 51.000 bis 52.000 Kindertageseinrichtungen zu rechnen. Im März 2010 wurden rund 16.000 einschlägig hochschulausgebildete Fachkräfte (DiplomSozialpädagoginnen und -Sozialpädagogen bzw. Diplom-Sozialarbeiterinnen und -Sozialarbeiter; Diplom-Pädagoginnen und -Pädagogen, Diplom-Heilpädagoginnen und -Heilpädagogen) in Kindertageseinrichtungen beschäftigt. Werden noch etwa 3.200 Lehrerinnen und Lehrer sowie Personen mit anderen Hochschulabschlüssen dazugerechnet, so waren im Jahr 2010 etwa 19.200 akademisch ausgebildete Personen in Kindertageseinrichtungen beschäftigt. Bis zum Jahr 2020 kämen nach den bisherigen Kapazitäten an Hochschulen rund 9.000 Personen hinzu (siehe Gutachten des Aktionsrats Bildung 2012), sodass ca. 28.200 Personen mit Hochschulabschluss in den Einrichtungen arbeiten würden. Bei 51.000 bis 52.000 Kindertageseinrichtungen würden somit zusätzlich zu den heutigen Kapazitäten rund 23.000 Hochschulabsolventinnen und -absolventen benötigt.

Angesichts der in diesem Aufsatz skizzierten hohen öffentlichen Anforderungen an die frühpädagogischen Einrichtungen wird in der (Fach-)Öffentlichkeit in Frage gestellt, ob die bisherige Ausbildung von Erzieherinnen und Erziehern - besonders aber die Ausbildung von Kinderpflegerinnen und -pflegern - hinreichend auf die heutige fachliche Arbeit in frühpädagogischen Einrichtungen, speziell im Hinblick auf die Bildungsförderung der Kinder, vorbereitet. Vor diesem Hintergrund wird einerseits die Akademisierung der Ausbildung gefordert, andererseits werden die besonderen Chancen einer reformierten fachschulischen Ausbildung betont.

Fachschulische Ausbildung zur Erzieherin bzw. zum Erzieher: Generell wird von den Fachverbänden, den Trägern wie auch von der Politik für eine Beibehaltung der bisherigen Breitbandausbildung auf Fachschulniveau plädiert (vgl. z.B. Jugendministerkonferenz (JMK) 2005; Langenmayr 2005; Rudolph 2010). Breitbandausbildung meint dabei, dass die fachschulische Ausbildung nicht nur auf die Arbeit in frühpädagogischen Einrichtungen vorbereitet, sondern auf die gesamte institutionelle und außerinstitutionelle Arbeit in den verschiedenen sozialpädagogischen Feldern mit Kindern und Jugendlichen im Alter von null bis 27 Jahren. Generell wird davon ausgegangen, dass eine „Spezialausbildung“ für den frühpädagogischen Bereich die Beschäftigungschancen des Erziehungspersonals deutlich schmälern würde, obwohl die Mehrheit der Erzieherinnen und Erzieher in Kindertageseinrichtungen beschäftigt ist. Allerdings wird ebenso die Notwendigkeit gesehen, innerhalb einer breiteren Ausbildung spezifische Schwerpunkte zu setzen (z.B. auf die Arbeit mit unter dreijährigen Kindern oder auf die Einrichtungsleitung) und ggf. auch berufsbegleitend (vgl. z.B. Bundesarbeitsgemeinschaft der Landesjugendämter 2009; Rudolph 2010). Angesichts der Unterschiedlichkeit der verschiedenen Arbeitsfelder in Kinder- und Jugendhilfe ist eine solche Spezialisierung auf den Bereich der frühkindlichen Bildung sinnvoll (siehe dazu die Empfehlungen des Aktionsrats Bildung (2012)).

Von verschiedenen Seiten werden auch bundesweite fachliche Mindeststandards und eine stärkere Vereinheitlichung der fachschulischen Ausbildung angemahnt (vgl. z.B. Rudolph 2010). Einen wichtigen Beitrag hierzu dürfte auch der Beschluss der Jugend- und Familienministerkonferenz vom 14. Dezember 2010 - gleichlautend der Beschluss der Kultusministerkonferenz (KMK) vom 16. September 2010 - leisten, den Gemeinsamen Orientierungsrahmen „Bildung und Erziehung in der Kindheit“ zustimmend zur Kenntnis 
zu nehmen (vgl. JFMK 2010). Dieser soll eine Grundlage für die Weiterentwicklung der Aus- und Weiterbildungsgänge an Fachschulen, Fachakademien und Hochschulen im Bereich der Bildung und Erziehung in der Kindheit bieten. Das Ziel der fachschulischen Ausbildung, die Befähigung, Erziehungs- und Bildungsaufgaben sowie Betreuungsaufgaben übernehmen zu können und in allen sozialpädagogischen Bereichen selbstständig und eigenverantwortlich tätig zu sein, wird vom Orientierungsrahmen bekräftigt. Die Bildung von Ausbildungsschwerpunkten nach Tätigkeitsfeld ist hierbei zugelassen.

Aktuell findet eine breite Diskussion über die Verbindung von fachschulischer und hochschulischer Ausbildung statt. Eine Vielzahl von Reformvorstellungen bezieht sich auf die Durchlässigkeit von fachschulischer und hochschulischer Ausbildung. Die während der fachschulischen Ausbildung erworbenen Kompetenzen sollen - falls die formalen Voraussetzungen erfüllt sind - auf ein Hochschulstudium angerechnet werden. Die JFMK (2010) und die KMK wollen in diesem Zusammenhang ermöglichen, bis zu 50 Prozent eines Hochschulstudiums durch außerhalb eines Hochschulstudiums erworbene Kenntnisse und Fähigkeiten zu ersetzen.

Berufsfachschulische Ausbildung zur Kinderpflegerin bzw. zum Kinderpfleger: Angesichts der Anforderungen an die Qualifikationen des pädagogischen Personals ist zu fordern, keine Neueinstellungen von Mitarbeiterinnen und Mitarbeitern im Gruppendienst in frühpädagogischen Einrichtungen ohne mindestens einen Fachschulabschluss vorzunehmen (vgl. z.B. Langenmayr 2005; Deutscher Berufsverband für Soziale Arbeit (DBSH) 2009). Damit würden Kinderpflegerinnen und -pfleger sowie Sozialassistentinnen und -assistenten keinen Eingang mehr in dieses Berufsfeld finden. Dabei muss aber zumindest berücksichtigt werden, dass die bereits in der Praxis tätigen Kinderpflegerinnen und -pfleger und Sozialassistentinnen und -assistenten bzw. die derzeit in der berufsfachschulischen Ausbildung befindlichen Personen systematisch weiterqualifiziert werden müssen, um ein höheres Qualifikationsniveau zu erreichen. Bereits heute verfügt die Mehrheit der Kinderpflegerinnen und -pfleger mindestens über einen mittleren Schulabschluss: 57,7 Prozent der derzeit als Kinderpflegerinnen bzw. Kinderpfleger in Kindertageseinrichtungen Beschäftigten verfügen über den mittleren Schulabschluss, die Fachhochschulreife oder die allgemeine oder fachbezogene Hochschulreife (vgl. Fuchs-Rechlin 2010). Insofern werden die Chancen als gut angesehen, die Ausbildung von Unterstützungspersonal wie Kinderpflegerinnen und -pflegern oder Sozialassistentinnen und -assistenten formal auf die Ebene der Fachschulen anzuheben.

Fort- und Weiterbildung: Die gegenwärtige Diskussion schließt nicht nur die Ausbildung an den Fachschulen für Sozialpädagogik oder an den Hochschulen mit ein; sie berücksichtigt ebenso die bereits im Feld tätigen Personen mit Abschlüssen an Fachschulen bzw. Berufsfachschulen.

Bedarf bei der Hochschulausbildung: Mehrere Gründe sprechen für eine Akademisierung der Ausbildung des frühpädagogischen Personals. Ohne Anspruch auf Vollständigkeit können drei Motivationen unterschieden werden, wobei die erste - vor dem Hintergrund der frühpädagogischen Einrichtungen als Bildungseinrichtungen - als zentral und die beiden weiteren als zusätzliche Begründungen anzusehen sind: (1) Förderung der kindlichen Entwicklung und Bildung: Eine Auseinandersetzung mit theoretischem, abstraktem und wissenschaftlichem Wissen - die bisher von Experten beim frühpädagogischen Personal häufig 
als fehlend erachtet wird - ist für die Förderung der Kinder unerlässlich. In den letzten Jahren ist zudem die Komplexität der Anforderungen an das Fachpersonal im Hinblick auf die Förderung der heterogener gewordenen Kindergruppen gestiegen; der Umgang mit Bildungsplänen und Übergängen sowie die Verzahnung mit der Grundschule verlangen nach höheren Qualifikationen des Fachpersonals. (2) Wettbewerbsfähigkeit und soziale Stellung der Fachkräfte: Durch die Akademisierung der Ausbildung soll die Tätigkeit im frühpädagogischen Bereich attraktiver und der Beruf gesellschaftlich aufgewertet werden, nicht zuletzt durch eine bessere Bezahlung. Damit ist auch eine Erhöhung der Attraktivität des Berufs für Männer verbunden. Der frühpädagogische Aufgabenbereich muss hoch motivierte und erfolgreiche Studierende anziehen und Absolventinnen und Absolventen ermuntern, für den Berufseinstieg ihr Fachgebiet anstelle anderer und besser vergüteter Berufsfelder zu wählen. (3) Professionelle Steuerung: Gut ausgebildetes Personal benötigt weniger Vorgaben für eine qualitativ hochwertige Arbeit und ist in der Lage, vielfältige Tätigkeiten, speziell im Hinblick auf Management- und Leitungsaufgaben, professionell auszuführen.

Nicht zuletzt befördert durch den Bologna-Prozess (vgl. v. Behr/Diller/Schelle 2009) gibt es seit dem Jahr 2004 eine rasante Entwicklung, in der frühpädagogische Bachelorstudiengänge in der Regel an (Fach-)Hochschulen sowie in Baden-Württemberg an pädagogischen Hochschulen eingerichtet wurden. Die Vielfalt dieser Studiengänge in struktureller und inhaltlicher Hinsicht ist enorm. Viernickel (2008) zählt für März 2008 über 55 Standorte mit knapp 60 umgesetzten oder in Planung befindlichen Studiengängen im frühpädagogischen Bereich. Pasternack und Schulze (2010) zählen im Jahr 2010 bereits 67 frühpädagogische Studienangebote. Werden frühpädagogisch affine und Kita-Management-Studiengänge hinzuaddiert, kommen die Autoren auf 78 Angebote. Die Robert Bosch Stiftung (2011) nennt nur ein Jahr später bereits 90 Studiengänge, einschließlich solcher, die dem Management von Kindertagesstätten zuzuordnen sind. Die weitgehend unkoordinierte Entwicklung von frühpädagogischen Studiengängen an meistens sozialpädagogisch orientierten Hochschulen, teilweise auch an Universitäten sowie in Baden-Württemberg an den pädagogischen Hochschulen wirft eine breite Palette von Problemen auf, die vor einer fachlich sinnvollen Weiterentwicklung der hochschulischen Ausbildung gelöst werden müssen.

Ein erster Problemkreis betrifft die Ausrichtung der hochschulischen Ausbildung: Soll es sich, wie bei der Ausbildung zur Erzieherin bzw. zum Erzieher, um eine Breitbandausbildung handeln (vgl. z.B. DBSH 2009; Arbeitsgemeinschaft für Kinder- und Jugendhilfe (AGJ) 2010) oder soll sie speziell auf das Fachpersonal für die Arbeit in Kindertageseinrichtungen ausgerichtet sein? Der Aktionsrat Bildung (2012) empfiehlt hier angesichts des gegenwärtigen Bedarfs an Fachkräften für die frühkindliche Bildung und der spezifischen Anforderungen der Erziehungs-, Bildungs- und Betreuungsarbeit in diesem Bildungsbereich eine Spezialisierung der Ausbildung auf die Arbeit in Kindertageseinrichtungen.

Ein zweiter Problemkreis bezieht sich auf den Arbeitsschwerpunkt von Absolventinnen und Absolventen akademischer Studiengänge: Sollen sie für die direkte Arbeit mit Kindern qualifiziert werden oder soll der Schwerpunkt auf Leitungsaufgaben liegen? Angesichts der Tatsache, dass in diesem Jahrzehnt insgesamt nur ein eher geringer Anteil des Fachpersonals akademisch ausgebildet werden kann bzw. bis 2020 einen einschlägigen akademischen Abschluss aufweisen wird, empfiehlt der Aktionsrat Bildung (2012) eine Schwerpunktsetzung der akademischen Ausbildungsgänge auf Leitungsfunktionen, wo- 
durch Anpassungen in den Ausbildungscurricula an den Hochschulen erforderlich wären. Im Mittelpunkt sollte die pädagogische Führung im Sinne des Vorlebens eines guten Rollenmodells, Teambildungsaufgaben, Supervision, Coaching und Anleitung sowie Vermittlung von Fachwissen an das Einrichtungsteam stehen. Die Empfehlung des Aktionsrats Bildung (2012), den Schwerpunkt der Hochschulausbildung auf Leitungsfunktionen zu legen, bedeutet auch die Übernahme unterschiedlicher Funktionen in einer Kindertageseinrichtung von auf unterschiedlichen Niveaus ausgebildetem Personal. Dazu müssen entsprechende Modelle der Arbeitsaufteilung gezielt entwickelt und in der Ausbildung auf den verschiedenen Ebenen berücksichtigt werden.

Der dritte Problemkreis thematisiert die notwendigen Praxisanteile in der hochschulischen Ausbildung. Welche Rolle spielt der Lernort Praxis in der Hochschulausbildung und wie umfangreich soll er sein? Verbindungen zwischen dem Lernort Praxis und dem Lernort Hochschule wie auch dem Lernort Fachschule sollten nach den Empfehlungen des Aktionsrats Bildung (2012) in Zukunft systematisch angelegt und curricular ausformuliert sein (vgl. Diskowski 2011).

Der vierte Problemkreis betrifft die Klientel für die hochschulische Ausbildung. Zunächst einmal bestehen gute Chancen, nicht nur Schulabgängerinnen und Schulabgänger, sondern ebenso bereits im Feld tätige Personen für das Hochschulstudium zu gewinnen. Erzieherinnen und Erzieher besitzen zu einem hohen Anteil die (Fach-)Hochschulreife und haben damit die formale Voraussetzung für ein Studium. Darüber hinaus liegt eine hohe Akzeptanz für eine berufsbegleitende Hochschulausbildung vor. Insgesamt empfiehlt der Aktionsrat Bildung (2012) deshalb, besondere Anstrengungen zu unternehmen, bereits im frühpädagogischen Feld arbeitende Personen für ein (berufsbegleitendes) Hochschulstudium zu gewinnen.

Der fünfte Problemkreis bezieht sich vor allem auf die Universitäten und die Frage, ob es überhaupt genügend einschlägiges, an den Universitäten frühpädagogisch qualifiziertes (promoviertes) Personal gibt, um die wissenschaftliche und forschungsbasierte Ausbildungssituation an Hochschulen zu sichern. Nachdem der frühpädagogische Erziehungs-, Bildungs- und Betreuungsbereich an den Universitäten jahrzehntelang vernachlässigt wurde - bis vor Kurzem gab es in Deutschland nur vier Lehrstühle für Frühpädagogik -, bedarf es dringend des Ausbaus von Forschungs- und Ausbildungsstrukturen an Universitäten. Der Aktionsrat Bildung (2012) fordert deswegen die Einrichtung weiterer frühpädagogischer Professuren und Lehrstühle.

Nach Ansicht des Aktionsrats Bildung (2012) besteht aber keine Notwendigkeit, das gesamte Personal in frühpädagogischen Einrichtungen auf Hochschulebene auszubilden. Als eine Möglichkeit des Verhältnisses von Fach- und Hochschulen bzw. der Weiterentwicklung von Fachschulen nennt Pasternack (2008) ein Institutionentransfermodell: Einzelne Fachschulen oder Fachschulteile werden nach einem erfolgreichen Organisationsentwicklungsprozess in Berufsakademien oder Fachhochschulen integriert. 


\section{Zusammenfassung der zentrale Empfehlungen des Aktionsrats Bildung}

Der Aktionsrat Bildung (2012) versteht Kindertageseinrichtungen in der Trias von Erziehung, Bildung und Betreuung primär als Bildungseinrichtungen. Vor diesem Hintergrund spricht er sich vor allem für die folgenden Handlungsempfehlungen aus:

1. Zur Professionalisierung des Fachpersonals in der Frühpädagogik sollte ein koordiniertes Gesamtkonzept für Aus-, Weiter- und Fortbildung auf verschiedenen Ebenen entwickelt werden. Ziel muss es sein, nicht auf einzelne Ausbildungsgänge auf Fachoder Hochschulebene zu fokussieren, sondern in einem Gesamtkonzept das frühpädagogische Arbeitskräftepotenzial insgesamt zu professionalisieren. Dazu gehört auch, die Durchlässigkeit der verschiedenen Ausbildungsgänge sicherzustellen.

2. Die Hochschulstudiengänge für das pädagogische Personal in der frühkindlichen Bildung sollten deutschlandweit vereinheitlicht werden und sich auf Erziehungs-, Bildungs- und Betreuungsarbeit in Kindertageseinrichtungen spezialisieren. Die hochschulischen Ausbildungscurricula sollten sich auf die Vermittlung von professionellen Handlungskompetenzen beziehen. Dabei sollte auch besonderer Wert auf eine systematische und curricular ausformulierte Abstimmung mit dem Lernort „Praxis“ gelegt werden. Die hochschulische Ausbildung sollte dabei vor allem auf die Übernahme von Leitungsfunktionen - speziell im Hinblick auf Teambildungsaufgaben, pädagogische Führung, Supervision, Coaching und Anleitung sowie Vermittlung von Fachwissen an das Einrichtungsteam - qualifizieren.

3. Um die große Motivation im Hinblick auf eine höhere Qualifizierung von bereits in Kindertageseinrichtungen tätigen Erzieherinnen und Erziehern mit Fachschulausbildung zu nutzen, sollten berufsbegleitende frühpädagogische Hochschulstudiengänge ausgeweitet werden.

4. Bis zum Jahr 2020 sollte in jeder Kindertageseinrichtung mindestens eine auf Hochschulebene einschlägig ausgebildete Fachkraft - zentral in der Einrichtungsleitung beschäftigt sein. Um diese Zielmarke zu erreichen, müssen über die bisherigen Kapazitäten an Hochschulen hinaus bis zum Jahr 2020 weitere rund 23.000 Fachkräfte an Hochschulen ausgebildet werden. Dies würde etwa eine Verdoppelung bis Verdreifachung der bestehenden Ausbildungskapazitäten bedeuten.

5. Um die Attraktivität der Hochschulausbildung und des Verbleibs im frühpädagogischen Feld zu steigern, wird empfohlen, die Vergütung für die Absolventinnen und Absolventen der frühpädagogischen Hochschulstudiengänge auf das Niveau der Vergütung für Absolventinnen und Absolventen vergleichbarer Studiengänge außerhalb des frühpädagogischen Bereichs anzuheben.

6. Die fachschulische Ausbildung kann zwar weiterhin als Breitbandausbildung angelegt sein, sollte aber eine dezidierte Schwerpunktbildung in der Erziehungs-, Bildungsund Betreuungsarbeit in Kindertageseinrichtungen ermöglichen. Die Ausbildungscurricula sollten sich ebenfalls auf die Vermittlung von professionellen Handlungskompetenzen beziehen. Die unterschiedlichen, auf Fachschul- und Hochschulebene zu erreichenden Kompetenzniveaus müssen weiter ausdifferenziert und transparent gemacht werden. 
7. Verpflichtende Fort- und Weiterbildungen des pädagogischen Personals in Kindertageseinrichtungen sollten als ein selbstverständlicher Standard betrachtet werden. Um darüber hinaus Anreize zur beruflichen Weiterentwicklung zu schaffen, sollte es dem in der Praxis tätigen frühpädagogischen Personal ermöglicht werden, über ein systematisches Weiterbildungsprogramm einen höheren Fachkräftestatus zu erreichen, ohne ein Hochschulstudium aufnehmen zu müssen. Dieses Weiterbildungsprogramm sollte in Verbindung mit den Hochschulen entwickelt und umgesetzt werden; der erfolgreiche Abschluss sollte zertifiziert werden und zu einer Verbesserung in der Vergütung führen; die Teilnahme sollte staatlich gefördert werden.

8. Mittelfristig sollte nur noch fachschulisch und hochschulisch ausgebildetes Personal in Kindertageseinrichtungen arbeiten, d.h., es sollte mittelfristig auf Neueinstellungen von Kinderpflegerinnen und Kinderpflegern sowie Sozialassistentinnen und Sozialassistenten verzichtet werden. Die bereits jetzt in der Praxis tätigen Kinderpflegerinnen und Kinderpfleger bzw. die derzeit in der berufsfachschulischen Ausbildung befindlichen Personen sollten systematisch weiterqualifiziert werden, um ein höheres Qualifikationsniveau zu erreichen. Personal, das in den frühpädagogischen Einrichtungen unterstützende Funktionen wahrnimmt, sollte - vor allem unter Bildungsgesichtspunkten - zukünftig auf einem höheren formalen Niveau ausgebildet werden.

9. Nachdem der frühpädagogische Erziehungs-, Bildungs- und Betreuungsbereich an den Universitäten jahrzehntelang vernachlässigt wurde, müssen dringend Forschungsund Ausbildungsstrukturen ausgebaut werden, um entsprechend wissenschaftlich qualifiziertes Personal für die Hochschulen, aber auch für die Fachschulen zur Verfügung stellen zu können. Dazu muss die Anzahl an Professuren für Frühpädagogik deutlich erhöht werden. Die Förderung des wissenschaftlichen Nachwuchses im frühpädagogischen Bereich wird als eine gesellschaftliche Aufgabe angesehen, bei der sich auch die Stiftungen engagieren sollten.

Das Ziel, bis zum Jahr 2020 in allen Kindertageseinrichtungen mindestens eine akademisch ausgebildete Fachkraft (bevorzugt in Leitungsfunktionen) zu beschäftigen, erfordert, über die gegenwärtig vorhandenen hochschulischen Kapazitäten hinaus, eine erhebliche zusätzliche Anzahl von Fachkräften auf Hochschulniveau auszubilden. Der Aktionsrat Bildung (2012) hat dazu in seinem Gutachten zwei detaillierte Szenarien entwickelt.

\section{Literatur}

Aktionsrat Bildung (2012). Professionalisierung in der Frühpädagogik. Qualifikationsniveau und -bedingungen des Personals in Kindertagesstätten. Gutachten. vbw. Münster: Waxmann (herausgegeben von der Vereinigung der Bayerischen Wirtschaft e.V. (vbw)).

Arbeitsgemeinschaft für Kinder- und Jugendhilfe (AGJ) (2010). Frühpädagogische Studiengänge im Spannungsfeld von Spezialisierung und Generalisierung. Positionspapier der Arbeitsgemeinschaft für Kinder- und Jugendhilfe. http://www.agj.de/pdf/5/Fruehpaedagogik.pdf [Stand: 2011-10-15].

Autorengruppe Bildungsberichterstattung (Hrsg.) (2010). Bildung in Deutschland 2010. Ein indikatorengestützter Bericht mit einer Analyse zu Perspektiven des Bildungswesens im demografischen Wandel. Bielefeld: Bertelsmann.

Barnett, W. S. (2008). Preschool education and its lasting effects: Research and policy implications. Boulder/Tempe: Education and the Public Interest Center and Education Policy Unit. http://www. nieer.org/resources/research/Preschool LastingEffects.pdf [Stand: 2011-09.15]. 
Baumert, J./Kunter, M. (2006). Stichwort: Professionelle Kompetenz von Lehrkräften. Zeitschrift für Erziehungswissenschaft, 9, 4, S. 469-520.

Behr, A. v./Diller, A./Schelle, R. (2009). Die Ausbildung der Erzieherinnen - aktuelle Entwicklungen und Herausforderung. Recht der Jugend und des Bildungswesens. Zeitschrift für Schule, Berufsbildung und Jugenderziehung, 57, 1, S. 146-158.

Blok, H./Fukkink, R. G./Gebhardt, E. C./Leseman, P. P. M. (2005). The relevance of delivery mode and other program characteristics for the effectiveness of early childhood intervention with disadvantaged children. International Journal of Behavioral Development, 29, 1, S. 35-47.

Bundesarbeitsgemeinschaft der Freien Wohlfahrtspflege (BAGFW) (2011). Ansätze und Überlegungen zum Umgang mit der Fachkräftesituation in Kindertageseinrichtungen. http://www.bagfw.de/ uploads/tx_twpublication/Fachkr\%C3\%A4fteentwicklung_in_Kindertageseinrichtungen_08.03.2011. pdf [Stand: 2011-10-15].

Bundesarbeitsgemeinschaft der Landesjugendämter (2009). Fachliche Empfehlungen zur Qualität der Bildung, Erziehung und Betreuung der unter Dreijährigen in Kindertageseinrichtungen und Kindertagespflege. http://www.bagljae.de/Stellungnahmen/107_Qualitaet\%20der\%20Bildung,\%20 Erziehung, \%20Betreuung\%20unter\%203jaehrige_2009.pdf [Stand: 2011-10-04].

Burchinal, M./Vandergrift, N./Pianta, R./Mashburn, A. (2010). Threshold analysis of association between child care quality and child outcomes for low income children in pre-kindergarten programs. Early Childhood Research Quarterly, 25, 2, S. 166-176.

Camilli, G./Vargas, S./Ryan, S./Barnett, W. S. (2010). Meta-analysis of the effects of early education interventions on cognitive and social development. Teachers College Record, 112, 3, S. 579-620.

Dearing, E./McCartney, K./Taylor, B. A. (2009). Does higher quality early child care promote lowincome children's math and reading achievement in middle childhood? Child Development, 80, 5, S. 1329-1349.

Deutscher Berufsverband für Soziale Arbeit (DBSH) (2009). Qualifizierung, Nachhaltigkeit und Akademisierung. Der DBSH setzt sich für eine umfassende Verbesserung der Qualifikation, Arbeitsbedingungen und Bezahlung von ErzieherInnen ein. http://www.dbsh.de/html/berufspolitik.html [Stand: 2011-10-04].

Deutscher Verein für öffentliche und private Fürsorge (2011). Eckpunktepapier des Deutschen Vereins $z u$ den Herausforderungen beim Ausbau der Kindertagesbetreuung für Kinder unter drei Jahren. http://www.deutscher-verein.de/05empfehlungen/empfehlungen_archiv/2010/pdf/DV\%2002-11_ Eckpunktepapier_Ausbau\%20KTB.pdf [Stand: 2011-10-04].

Diskowski, D. (2008). Bildungspläne für Kindertagesstätten - ein neues und noch unbegriffenes Steuerungsinstrument. Zeitschrift für Erziehungswissenschaft, Sonderheft 11, S. 47-61.

Diskowski, D. (2011). Ist Akademisierung selbstverständlich Professionalisierung? Kopf und Bauch ein unbegriffener Zusammenhang in der Handlungskompetenz von Erzieherinnen. Theorie und Praxis der Sozialpädagogik, 4/2011, S. 48-52.

Early, D. M./Bryant, D. M./Pianta, R. C./Clifford, R. M./Burchinal, M. R./Ritchie, S./Howes, C./Barbarin, O. (2006). Are teachers' education, major, and credentials related to classroom quality and children's academic gains in pre-kindergarten? Early Childhood Research Quarterly, 21, 2, S. 174-195.

Early, D. M./Maxwell, K. L./Burchinal, M./Alva, S./Bender, R. H./Bryant, D./Cai, K./Clifford, R. M./ Ebanks, C./Griffin, J. A./Henry, G. T./Howes, C./Iriondo-Perez, J./Jeon, H.-J./Mashburn, A. J./ Peisner-Feinberg, E./Pianta, R. C./ Vandergrift, N. (2007). Teachers' education, classroom quality, and young children's academic skills: Results from seven studies of preschool programs. Child Development, 78, 2, S. 558-580.

Forschungsverbund Deutsches Jugendinstitut und Universität Dortmund (2007). Wie gehts im Job? KiTa-Studie der GEW. Frankfurt am Main: Gewerkschaft Erziehung und Wissenschaft.

Fuchs-Rechlin, K. (2009). Akademisierung in Kindertageseinrichtungen - Schein oder Sein? KOMDAT - Kommentierte Daten der Kinder- und Jugendhilfe, 12, 1, S. 18-19.

Fuchs-Rechlin, K. (2010). Die berufliche, familiäre und ökonomische Situation von Erzieherinnen und Kinderpflegerinnen. Sonderauswertung des Mikrozensus. Im Auftrag der Max-Traeger-Stiftung der $G E W$. Frankfurt am Main: Gewerkschaft Erziehung und Wissenschaft. http://www.gew.de/Binaries/ Binary71323/WEB\%20Mikrozensus.pdf [Stand: 2011-09-11]. 
Fukkink, R. G./Lont, A. (2007). Does training matter? A meta-analysis and review of caregiver training studies. Early Childhood Research Quarterly, 22, 3, S. 294-311.

Hall, J./Sylva, K./Melhuish, E./Sammons, P./Siraj-Blatchford, I./Taggart, B. (2009). The role of preschool quality in promoting resilience in the cognitive development of young children. Oxford Review of Education, 35, 3, S. 331-352.

Hasselhorn, M. (2005). Lernen im Altersbereich zwischen 4 und 8 Jahren: Individuelle Voraussetzungen, Entwicklung, Diagnostik und Förderung. In: Guldimann, T./Hauser, B. (Hrsg.), Bildung 4- bis 8jähriger Kinder. Münster: Waxmann, S. 77- 88.

Hasselhorn, M./Grube, D. (2008). Individuelle Voraussetzungen und Entwicklungsbesonderheiten des Lernens im Vorschul- und frühen Schulalter. Empirische Pädagogik, 22, 2, S. 113-126.

Havnes, T./Mogstad, M. (2011). No child left behind: Subsidized child care and children's long-run outcomes. American Economic Journal: Economic Policy, 3, 2, S. 97-129.

Heckman, J. J./Moon, S. H./Pinto, R./Savelyev, P./Yavitz, A. (2010). A new cost-benefit and rate of return analysis for the Perry Preschool Program: A summary. Bonn: Institut zur Zukunft der Arbeit (IZA Policy Paper No. 17).

Heisner, M. J./Lederberg, A. R. (2011). The impact of child development associate training on the beliefs and practices of preschool teachers. Early Childhood Research Quarterly, 26, 2, S. 227-236.

Helm, J. (2010): Das Bachelorstudium Frühpädagogik. Zugangswege - Studienzufriedenheit - Berufserwartungen. Ergebnisse einer Befragung von Studierenden. Eine Studie der Weiterbildungsinitiative Frühpädagogische Fachkräfte (WiFF). München: Deutsches Jugendinstitut.

Howes, C. (1997). Children's experiences in center-based child care as a function of teacher background and adult:child ratio. Merrill-Palmer Quarterly, 43, 3, S. 404-425.

Jugendministerkonferenz (JMK) (2005). Beschlüsse JMK am 12./13. Mai 2005 in München. Top 10 Aufgabenprofile und Qualifikationsanforderungen in den Arbeitsfeldern der Kinder- und Jugendhilfe. http://www.mbjs.brandenburg.de/media_fast/5527/TOP\%2010\%20-\%20Beschluss. 15475637. pdf [Stand: 2011-10-04].

Jugend- und Familienministerkonferenz (JFMK) (2008). Beschlüsse der JFMK-Sitzung am 29./30. Mai 2008 in Berlin. http://www.mbjs.brandenburg.de/sixcms/ detail.php/bb1.c.186500.de [Stand. 2011-10-05].

Jugend- und Familienministerkonferenz (JFMK) (2010). Umlaufbeschluss vom 14. Dezember 2010. Weiterentwicklung der Aus-, Fort- und Weiterbildung von Erzieherinnen und Erziehern; Gemeinsamer Orientierungsrahmen ,Bildung und Erziehung in der Kindheit". http://www.mbjs.brandenburg.de/media _fast $/ 5527 / 06-2010 \% 20 \mathrm{JFMK}-U m$ laufbeschluss $\% 20$ Gemeinsamer\%20Orientierungsrahmen.PDF, sowie die Anlage http:/www.mbjs.brandenburg.de/mehttp:/www.mbjs.brandenburg.de/media_fast/ 5527/06-2010\%20JFMK-Umlaufbeschluss\%20Gemeinsamer\%20Orientierungsrahmen.PDF/dia_fast/ 5527/Anlage\%20Gemeinsamer\%20Orientierungsrahmen \%20vom\%2016.PDF [Stand: 2011-10-04].

Jugend- und Familienministerkonferenz (JFMK) (2011). Beschlüsse JFMK 26./27. Mai 2011 in Essen. http:// www.jfmk.de/JFMK_AGJF/Beschl_sse_JFMK_26_27_05_2011/index.html [Stand: 2011-10-04].

Kelley, P./Camilli, G. (2007). The impact of teacher education on outcomes in center-based early childhood education programs: A meta-analysis. [NIEER Working Paper]. http://nieer.org/resources/ research/TeacherEd.pdf [Stand; 2011-07-04].

Kluczniok, K./Roßbach, H. G./Große, C. (2010). Fördermöglichkeiten im Kindergarten: Ein Systematisierungsversuch. In: Diller, A./Leu, H. R./Rauschenbach, T. (Hrsg.), Wie viel Schule verträgt der Kindergarten? Annäherung zweier Lernwelten. München: Deutsches Jugendinstitut, S. 133-152.

Kuger, S./Kluczniok, K. (2008). Prozessqualität im Kindergarten - Konzept, Umsetzung und Befunde. Zeitschrift für Erziehungswissenschaft, Sonderheft 11, S. 159-178.

Langenmayr, M. (2005). Quo vadis Erzieher/innen-Ausbildung? Acht Anfragen aus der Sicht der Fachschulen/Fachakademien für Sozialpädagogik. Kita spezial, Sonderheft 3/2005, S. 39-45.

Leseman, P. P. M. (2009). Die Wirkung qualitativ hochwertiger Betreuungs-, Bildungs- und Erziehungsangebote auf die frühkindliche Entwicklung. Zusammenfassung der Forschungsergebnisse. In: EACEA/Eurydice (Hrsg.), Frühkindliche Betreuung, Bildung und Erziehung in Europa: ein Mittel zur Verringerung sozialer und kultureller Ungleichheiten. Brüssel: Exekutivagentur für Bildung, Audiovisuelles und Kultur, S. 17-50. 
Mason, K.O./Duberstein, L. (1992). Consequences of child care for parent's well-being. In: Booth, A. (Hrsg.), Child care in the 1990s. Trends and consequences. Hillsdale, NJ: Erlbaum, S. 127-158

Oberhuemer, P./Schreyer, I. (2010). Kita-Fachpersonal in Europa. Ausbildungen und Professionsprofile. Opladen: Barbara Budrich.

Pasternack, P./Schulze, H. (2010). Die frühpädagogische Ausbildungslandschaft. Strukturen, Qualifikationsrahmen und Curricula. Gutachten für die Robert Bosch Stiftung Halle-Wittenberg: Martin-Luther-Universität Halle-Wittenberg, Institut für Hochschulforschung (HoF Arbeitsbericht 2‘10),

Rauschenbach, T./Schilling, M. (2010). Der U3-Ausbau und seine personellen Folgen. Empirische Analysen und Modellrechnungen. München: Deutsches Jugendinstitut (WIFF-Studie Nr. 1).

Robert Bosch Stiftung (Hrsg.) (2011). Qualifikationsprofile in Arbeitsfeldern der Pädagogik der Kindheit. Ausbildungswege im Überblick. Stuttgart: Robert Bosch Stiftung GmbH

Roßbach, H. G. (2004). Kognitiv anregende Lernumwelten im Kindergarten. Zeitschrift für Erziehungswissenschaft, 7. Jahrgang, Beiheft 3, S. 9-24.

Roßbach, H. G. (2005). Effekte qualitativ guter Betreuung, Bildung und Erziehung im frühen Kindesalter auf Kinder und ihre Familien. In: Sachverständigenkommission Zwölfter Kinder- und Jugendbericht (Hrsg.), Bd. 1: Bildung, Betreuung und Erziehung von Kindern unter sechs Jahren. München: Deutsches Jugendinstitut, S. 55-174.

Roßbach, H. G. (2008). Vorschulische Erziehung. In: Cortina, K. S./Baumert, J./Leschinsky, A./Mayer, K. U./Trommer, L. (Hrsg.), Das Bildungswesen in der Bundesrepublik Deutschland. Strukturen und Entwicklungen im Überblick. Reinbek bei Hamburg: Rowohlt, S. 283-323 (Völlig überarbeitete Neuausgabe).

Roßbach, H. G./Kluczniok, K./Kuger, S. (2008). Auswirkungen eines Kindergartenbesuchs auf den kognitiv-leistungsbezogenen Entwicklungsstand von Kindern. Zeitschrift für Erziehungswissenschaft, Sonderheft 11, S. 139-158.

Roßbach, H. G./Sechtig, J./Freund, U. (2010). Empirische Evaluation des Modellversuchs „Kindergarten der Zukunft in Bayern - KiDZ“. Ergebnisse der Kindergartenphase. Bamberg: University of Bamberg Press.

Rudolph, B. (2010). Anschlussfähige Bildungswege in der Ausbildung von Erzieherinnen und Erziehern. Ergebnisse einer Interviewstudie mit Leitungen von Fachschulen und Berufsfachschulen. Eine Studie der Weiterbildungsinitiative Frühpädagogische Fachkräfte (WiFF). München: Deutsches Jugendinstitut (WIFF Studien 2).

Sammons, P./Anders, Y./Sylva, K./Melhuish, E./Siraj-Blatchford, I./Taggart. B./Barreau, S. (2008). Children's cognitive attainment and progress in English primary schools during key stage 2: Investigating the potential continuing influences of pre-school education. Zeitschrift für Erziehungswissenschaft, Sonderheft 11, S. 179-198.

Schilling, M. (2011a). Die Zukunftsbranche Kinder- und Jugendhilfe - Personalbedarfe bis 2025 belaufen sich auf 333.000 Fachkräfte. KomDat - Kommentierte Daten der Kinder- und Jugendhilfe, 14 , $1-2$, S. $1-8$.

Schilling, M. (2011b). Fachkräftebedarf in Kindertageseinrichtungen bis zum Jahr 2020. URL: http:// www.aktionsrat-bildung.de/index.php?id=75.

Schweinhart, L. J./Montie, J./Xiang, Z./Barnett, W. S./Belfield, C. R./Nores, M. (2005). Lifetime effects. The High/Scope Perry Pre-School Study through age 40. Ypsilanti, MI: High/Scope Press.

Siraj-Blatchford, I./Sylva, K./Muttock, S./Gilden, R./Bell, D. (2002). Researching effective pedagogy in the early years. London: Department for Education and Skills (Research Report No. 356).

Statistisches Bundesamt (2009). Verdienste und Arbeitskosten. Verdienststrukturerhebung 2006 - Verdienste nach Berufen. Wiesbaden: Statistisches Bundesamt.

Statistisches Bundesamt (2011). Statistiken der Kinder- und Jugendhilfe. Kinder und tätige Personen in Tageseinrichtungen und in öffentlich geförderter Kindertagespflege am 01.03.2011. Wiesbaden: Statistisches Bundesamt.

Sylva, K./Melhuish, E. C./Sammons, P./Siraj-Blatchford, I./Taggart, B./Elliot, K. (2004). The effective provision of Pre-School Education Project - Zu den Auswirkungen vorschulischer Einrichtungen in 
England. In: Faust, G./Götz, M./Hacker, H./Roßbach, H. G. (Hrsg.), Anschlussfähige Bildungsprozesse im Elementar- und Primarbereich. Bad Heilbrunn: Julius Klinkhardt, S. 154-167.

Taggart, B./Sylva, K./Siraj-Blatchford, I./Melhuish, E. C./Sammons, P./Walker-Hall, J. (2000). The effective provision of Pre-School Education (EPPE) Project: Technical paper 5 - Characteristics of the centres in the EPPE sample: Interviews. London: Department for Education and Employment/ Institute of Education, University of London.

Thole, W. (2010). Die pädagogischen MitarbeiterInnen in Kindertageseinrichtungen. Professionalität und Professionalisierung eines pädagogischen Arbeitsfeldes. Zeitschrift für Pädagogik, 56, 2, S. 206-222.

Tietze, W./Roßbach, H. G./Grenner, K. (2005). Kinder von 4 bis 8 Jahren. Zur Qualität der Erziehung und Bildung in Kindergarten, Grundschule und Familie. Weinheim: Beltz.

Vandell, D. L. (2011). Results of the NICHD-Study: Effects of early child care are found at age 15. Vortrag auf der Fachtagung „Qualität und Effekte frühkindlicher Bildung und Betreuung: ein internationaler Vergleich“ der Robert Bosch Stiftung und des Lehrstuhls für Elementar- und Familienpädagogik der Otto-Friedrich-Universität Bamberg am 17./18.11.2011 in Berlin.

Vandell, D.L./Wolfe, B. (2000). Child care quality: Does it matter and does it need to be improved? University of Wisconsin-Madison, Institute for Research on Poverty. http://www.irp.wisc.edu/ publications/sr/pdfs/sr78.pdf [Stand: 2012-06-02],

Viernickel, S. (2008). Reformmodelle für die Ausbildung des frühpädagogischen Fachpersonals. Zeitschrift für Erziehungswissenschaft, Sonderheft 11, S. 123-138.

Whitebook, M. (2003). Early education quality: Higher teacher qualifications for better learning environments - A review of the literature. Summary version http://nccic.acf.hhs.gov/node/27643 [Stand: 2012-07-07].

Eingereicht am/Submitted on: 03.05.2012

Angenommen am/Accepted on: 23.06,2012

Anschriften derAutoren/Addresses of the authors:

Prof. Dr. Hans-Günther Roßbach

Otto-Friedrich-Universität Bamberg

Lehrstuhl für Elementar- und Familienpädagogik

Markusstraße 8a

96047 Bamberg

Deutschland/Germany

Prof. Dr. Dr. h. c. Hans-Peter Blossfeld

Otto-Friedrich-Universität Bamberg

Lehrstuhl für Soziologie I

Wilhelmsplatz 3

96047 Bamberg

Deutschland/Germany

E-Mail: hans-peter.blossfeld@uni-bamberg.de hans-guenther.rossbach@uni-bamberg.de 